\title{
Reduced sensitivity of SARS-CoV-2 variant Delta to antibody neutralization
}

https://doi.org/10.1038/s41586-021-03777-9

Received: 26 May 2021

Accepted: 29 June 2021

Published online: 8 July 2021

Check for updates

\author{
Delphine Planas ${ }^{1,2}$, David Veyer ${ }^{3,4}$, Artem Baidaliuk ${ }^{5}$, Isabelle Staropoli', \\ Florence Guivel-Benhassine ${ }^{1}$, Maaran Michael Rajah ${ }^{1,6}$, Cyril Planchais $^{7}$, Françoise Porrot ${ }^{1}$, \\ Nicolas Robillard ${ }^{4}$, Julien Puech ${ }^{4}$, Matthieu Prot ${ }^{5}$, Floriane Gallais ${ }^{8,9}$, Pierre Gantner ${ }^{8,9}$, \\ Aurélie Velay ${ }^{8,9}$, Julien Le Guen ${ }^{10}$, Najiby Kassis-Chikhani ${ }^{11}$, Dhiaeddine Edriss ${ }^{4}$, \\ Laurent Belec ${ }^{4}$, Aymeric Seve ${ }^{12}$, Laura Courtellemont ${ }^{12}$, Hélène Péré ${ }^{3}$, Laurent Hocqueloux ${ }^{12}$, \\ Samira Fafi-Kremer ${ }^{8,9}$, Thierry Prazuck ${ }^{12}$, Hugo Mouquet ${ }^{7}$, Timothée Bruel ${ }^{1,2,14} \bowtie$, \\ Etienne Simon-Lorière ${ }^{5,14}$, Felix A. Rey ${ }^{13,14}$ \& Olivier Schwartz ${ }^{1,2,14 凶}$
}

\begin{abstract}
The SARS-CoV-2 B.1.617 lineage was identified in October 2020 in India ${ }^{1-5}$. Since then, it has become dominant in some regions of India and in the UK, and has spread to many other countries ${ }^{6}$. The lineage includes three main subtypes (B1.617.1, B.1.617.2 and B.1.617.3), which contain diverse mutations in the N-terminal domain (NTD) and the receptor-binding domain (RBD) of the SARS-CoV-2 spike protein that may increase the immune evasion potential of these variants. B.1.617.2-also termed the Delta variantis believed to spread faster than other variants. Here we isolated an infectious strain of the Delta variant from an individual with COVID-19 who had returned to France from India. We examined the sensitivity of this strain to monoclonal antibodies and to antibodies present in sera from individuals who had recovered from COVID-19 (hereafter referred to as convalescent individuals) or who had received a COVID-19 vaccine, and then compared this strain with other strains of SARS-CoV-2. The Delta variant was resistant to neutralization by some anti-NTD and anti-RBD monoclonal antibodies, including bamlanivimab, and these antibodies showed impaired binding to the spike protein. Sera collected from convalescent individuals up to 12 months after the onset of symptoms were fourfold less potent against the Delta variant relative to the Alpha variant (B.1.1.7). Sera from individuals who had received one dose of the Pfizer or the AstraZeneca vaccine had a barely discernible inhibitory effect on the Delta variant. Administration of two doses of the vaccine generated a neutralizing response in $95 \%$ of individuals, with titres three- to fivefold lower against the Delta variant than against the Alpha variant. Thus, the spread of the Delta variant is associated with an escape from antibodies that target non-RBD and RBD epitopes of the spike protein.
\end{abstract}

The SARS-CoV-2 Delta variant (B.1.617.2) has been detected in many countries. It has become predominant in the Indian state of Maharashtra and probably other Indian regions ${ }^{4}$, and represented $77 \%$ of the sequenced viruses circulating in the UK between 2 June and 9 June $2021^{6}$. It has been classified as a variant of concern (VOC) and is believed to be $60 \%$ more transmissible than the Alpha variant (B.1.1.7). Little is known about the sensitivity of the Delta variant to the humoral immune response. Recent reports have indicated that members of the B.1.617 lineage exhibit a reduced sensitivity to certain monoclonal and polyclonal antibodies compared to the Alpha variant $^{1-5,7-9}$.

\section{Isolation and characterization of the Delta variant}

We isolated the Delta variant from a nasopharyngeal swab of a symptomatic individual a few days after his return to France from India. The virus was amplified by two passages on Vero E6 cells. Sequences of the swab and the outgrown virus were identical, and identified the Delta

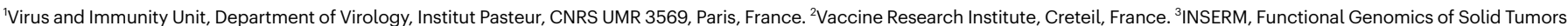
(FunGeST), Centre de Recherche des Cordeliers, Université de Paris and Sorbonne Université, Paris, France. ${ }^{4}$ Laboratoire de Virologie, Service de Microbiologie, Hôpital Européen Georges Pompidou, Assistance Publique des Hôpitaux de Paris, Paris, France. ${ }^{5} \mathrm{G} 5$ Evolutionary Genomics of RNA Viruses, Department of Virology, Institut Pasteur, Paris, France. ${ }^{6}$ Université de Paris, Sorbonne Paris Cité, Paris, France. ${ }^{7}$ Laboratory of Humoral Immunology, Department of Immunology, Institut Pasteur, INSERM U1222, Paris, France. ${ }^{8} \mathrm{CHU}$ de Strasbourg, Laboratoire de

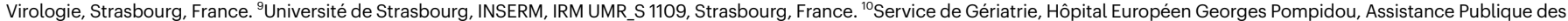

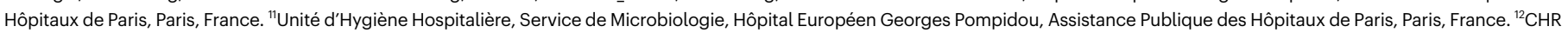
d'Orléans, Service de Maladies Infectieuses, Orléans, France. ${ }^{13}$ Structural Virology Unit, Department of Virology, Institut Pasteur, CNRS UMR 3569, Paris, France. ${ }^{14}$ These authors contributed

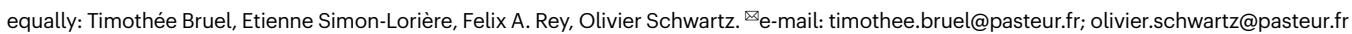



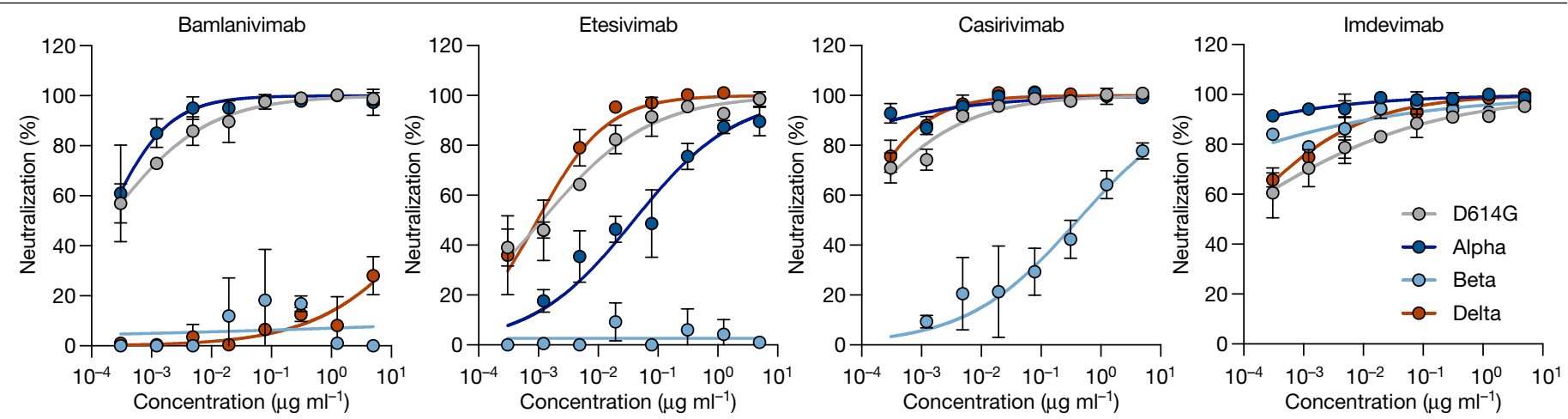

Fig. 1 Neutralization of the SARS-CoV-2 variants D614G, Alpha, Beta and Delta by therapeutic monoclonal antibodies. Neutralization curves of monoclonal antibodies. Dose-response analysis of neutralization of the D614G

strain and the Alpha, Beta and Delta variants by four therapeutic monoclonal antibodies (bamlanivimab, etesivimab, casirivimab and imdevimab). Data are mean \pm s.d. of four independent experiments.

variant (GISAID accession ID: EPI_ISL_2029113) (Extended Data Fig.1). When compared to the D614G strain (belonging to the basal B.1 lineage), which was used here as a reference, the spike protein contained eight mutations, including four mutations in the NTD (T19R, G142D, $\Delta 156-157$ and R158G), two in the RBD (L452R and T478K), one mutation close to the furin-cleavage site (P681R) and one in the S2 region (D950N) (Extended Data Fig. 1). This set of mutations was different from that observed in other members of the B.1.617 lineage and other VOCs (Extended Data Fig. 1). Viral stocks were titrated using S-Fuse reporter cells and Vero cells ${ }^{10,11}$. The viral titres were similar in the two target cells and reached $10^{5}-10^{6}$ infectious units per $\mathrm{ml}$. Large syncytia expressing the spike protein were observed in cells that were infected with the Delta variant (Extended Data Fig. 2). Future work will help to determine whether Delta is more fusogenic than other variants, as suggested here by the large size of Delta-induced syncytia.

\section{Phylogenetic analysis of the B.1.617 lineage}

To contextualize the isolate of the Delta variant reported here, we inferred a global phylogeny by subsampling the diversity of SARS-CoV-2 sequences available on the GISAID EpiCoV database (Extended Data Fig. 3). The B.1.617 lineage, which is divided into three sublineages according to the PANGO classification ${ }^{12}$, derives from the B.1 lineage (D614G). The three sublineages show multiple changes in the spike protein, including the L452R substitution in the RBD, which has already been observed in other variants such as B.1.429, and P681R. This substitution is located in the furin-cleavage site and may enhance the fusogenic activity of the spike protein ${ }^{13}$. The $\mathrm{E} 484 \mathrm{Q}$ substitution-which may be functionally similar to the antibody-escape mutation E484K that is found in the Beta and Gamma variants (B.1.351 and P.1, respectively)-is present in the B.1.617.1 and B.1.617.3 subtypes, and is likely to have reverted in the Delta sublineage, as it was present in a sequence (B.1.617) ancestral to the three sublineages ${ }^{14}$ (Extended Data Fig. 1). Whether the absence of $\mathrm{E} 484 \mathrm{Q}$, the presence of $\mathrm{T} 478 \mathrm{~K}$ or other changes in the spike protein or elsewhere may facilitate viral replication and transmissibility remains unknown. Notably, the B.1.617 lineage is not homogeneous, with multiple mutations that are fixed in a sublineage (for example, the spike protein mutations T19R, G142D or D950N) also being detected at lower frequencies in other sublineages. This may reflect founder effects or similar selective pressures acting on these emerging variants.

\section{Mutational changes in the Delta variant}

The locations of the spike protein mutations in the Delta variant showed a similar overall distribution to those that appeared in other VOCs. In particular, in addition to D614G, the D950N mutation mapped to the

trimer interface (Extended Data Fig. 4a), suggesting that this mutation may contribute to the regulation of spike protein dynamics, as shown for the D614G mutation ${ }^{13}$. As with other VOCs, some mutations in the Delta variant cluster in the NTD (Extended Data Fig. 4b). The 156-157 deletion and G158R mutation in the Delta variant map to the same surface as the 144 and 241-243 deletions in the Alpha and Beta (B.1.351) variants, respectively. The T19R mutation maps to a surface patch that has several mutations in the Alpha variant. These altered residues are found in the NTD 'supersite' that is targeted by most anti-NTD neutralizing antibodies ${ }^{15}$. In the RBD, mutations appearing in VOCs map to the periphery of the ACE2-binding surface (Extended Data Fig. 4c), suggesting that the virus accumulates mutations there to reduce or avoid recognition by antibodies while maintaining binding to ACE2. For example, the L452R mutation found in the Delta variant impairs neutralization by antibodies ${ }^{16}$ and is located at this periphery. The only mutation within the ACE2 patch is at location 501, which increases the affinity of the RBD for ACE2 and is also involved in antibody escape ${ }^{13}$. The T478K mutation in the RBD is unique to the Delta variant and falls within the epitope region of potent neutralizing monoclonal antibodies categorized as 'Class $1^{17}$ (Extended Data Fig. 4c). This mutation is close to the $\mathrm{E} 484 \mathrm{~K}$ mutation that facilitates antibody escape ${ }^{13}$. These observations prompted us to analyse the neutralization potential, against the Delta variant, of monoclonal antibodies and sera from convalescent individuals who had recovered from COVID-19 and from individuals who had been vaccinated against COVID-19.

\section{Neutralization of Delta by antibodies}

We assessed the sensitivity of the Delta variant to a panel of human monoclonal antibodies using the S-Fuse assay. We tested four clinically approved monoclonal antibodies that target the $\mathrm{RBD}^{18,19}$ (bamlanivimab (LY-CoV555), etesevimab (LY-CoV016), casirivimab (REGN10933) and imdevimab (REGN10987)), as well as eight anti-RBD (RBD-48, RBD-85, RBD-98 and RBD-109) and anti-NTD (NTD-18, NTD-20, NTD-69 and NTD71) monoclonal antibodies derived from convalescent individuals (C.P. et al., manuscript in preparation). Neutralizing anti-RBD monoclonal antibodies can be classified into four main categories ${ }^{17,20}$. RBD-48 and RBD- 85 belong to the first category (Class 1 ) and act by blocking the binding of the 'up' conformation of RBD to ACE $2^{17}$. The precise epitopes of RBD-98 and RBD-109 are not yet defined but overlap with those of RBD-48 and RBD-85. The anti-NTD antibodies bind to uncharacterized epitopes.

We measured the potency of the four therapeutic antibodies against the Delta variant and included as a comparison D614G (B.1) and the Alpha and Beta variants. The antibodies neutralized D614G with a half-maximal inhibitory concentration $\left(\mathrm{IC}_{50}\right)$ that ranged from $1.2 \times 10^{-3}$ to $6.5 \times 10^{-2} \mu \mathrm{g} \mathrm{ml}^{-1}$ (Fig.1). Etesivimab exhibited a 200 -fold increase in 


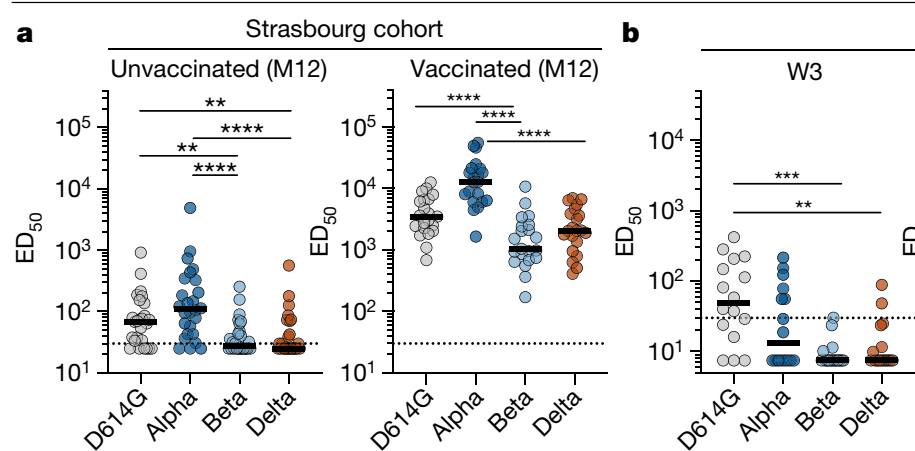

Fig. 2 | Sensitivity of the SARS-CoV-2 variants D614G, Alpha, Beta and Delta to sera from convalescent individuals and vaccinated individuals. Neutralization titres of the sera against the indicated viral isolates are expressed as $\mathrm{ED}_{50}$ values. a, Neutralizing activity of sera from the Strasbourg cohort of convalescent individuals ( $n=26$; left) and convalescent individuals who had been vaccinated ( $n=21$; right). Samples were collected at month 12 (M12) after the onset of symptoms. b, Neutralizing activity of sera from recipients of the Pfizer vaccine, sampled at week 3 (W3) after vaccination ( $n=16$; left) and week 8 (W8) after vaccination (week 5 after the second dose) ( $n=16$; right).c, Neutralizing activity of sera from recipients of the AstraZeneca vaccine, sampled at week 10 (W10) after vaccination $(n=23$; left $)$ and week 16 (W16) after vaccination (week 4 after the second dose) $(n=20$; right). The dotted line indicates the limit of detection $\left(E_{50}=30\right)$. Data are mean from two

Pfizer
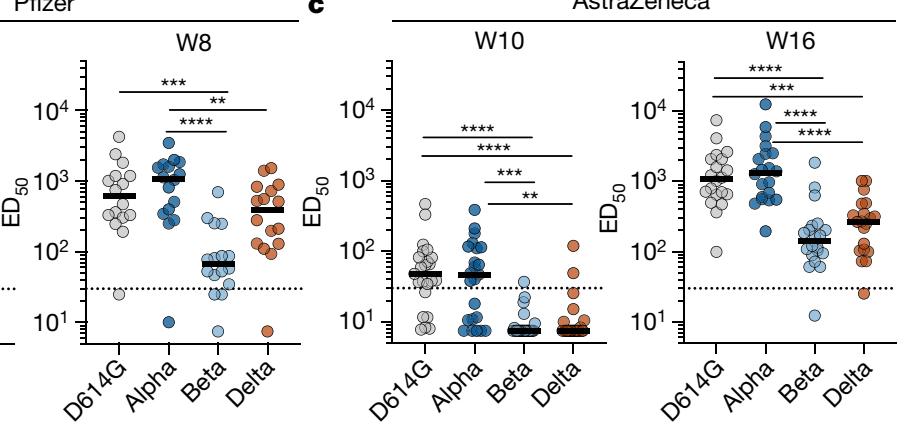

independent experiments. A two-sided Friedman test with Dunn's multiple comparison was performed between each of the viral strains. ${ }^{*} P<0.05$, ${ }^{* *} P<0.01,{ }^{* * *} P<0.001,{ }^{* * * *} P<0.0001$. Strasbourg cohort, unvaccinated (M12): D614G versus Beta, $P=0.0052$; D614G versus Delta, $P=0.0052$; Alpha versus Beta, $P<0.0001$; Alpha versus Delta, $P<0.0001$. Strasbourg cohort, vaccinated (M12): D614G versus Beta, $P<0.0001$; Alpha versus Beta, $P<0.0001$; Alpha versus Delta, $P<0.0001$. Pfizer (W3): D614G versus Beta, $P=0.0001 ; \mathrm{D} 614 \mathrm{G}$ versus Delta, $P=0.0013$. Pfizer (W8): D614G versus Beta, $P=0.0002$; Alpha versus Beta, $P<0.0001$; Alpha versus Delta, $P=0.0098$. AstraZeneca (W10): D614G versus Beta, $P<0.0001$; D614G versus Delta, $P<0.0001$; Alpha versus Beta, $P=0.0006$; Alpha versus Delta, $P=0.0056$. AstraZeneca (W16): D614G versus Beta, $P<0.0001$; D614G versus Delta, $P=0.0005$, Alpha versus Beta, $P<0.0001$; Alpha versus Delta, $P<0.0001$.

$\mathrm{IC}_{50}$ against the Alpha variant. As previously reported, bamlanivimab and etesivimab did not neutralize the Beta variant ${ }^{21}$. Bamlanivimab lost antiviral activity against the Delta variant, in line with previous results that showed that $L 452 \mathrm{R}$ is an escape mutation for this antibody ${ }^{16}$. Etesivimab, casirivimab and imdevimab remained active against the Delta variant (Fig. 1).

The four other anti-RBD monoclonal antibodies neutralized D614G. The IC $_{50}$ values of RBD-48 and RBD-98 were about 15 -100-fold higher for the Alpha variant than for D614G, whereas RBD-85 exhibited increased activity against the Alpha variant. Three monoclonal antibodies inhibited the Delta variant, whereas RBD-85 was inactive (Extended Data Fig. 5).

The four anti-NTD monoclonal antibodies were globally less efficient than anti-RBD monoclonal antibodies. They inhibited D614G with a high $\mathrm{IC}_{50}$ value (1-60 $\mathrm{g} \mathrm{m} \mathrm{ml}^{-1}$ ) (Extended Data Fig. 5). Three anti-NTD antibodies lost activity against the Alpha and Delta variants, whereas the fourth (NTD-18) inhibited the two variants to some extent. Thus, the Delta variant escapes neutralization by some antibodies that target the RBD or NTD.

We examined by flow cytometry the binding of each monoclonal antibody to Vero cells infected with the different variants, and used radar plots to show the binding of all antibodies tested (Extended Data Fig. 6). D614G was recognized by the 12 monoclonal antibodies tested. The Alpha and Delta variants were recognized by nine and by seven monoclonal antibodies, respectively. Bamlanivimab no longer bound to the Delta variant. We also analysed the binding of the 12 monoclonal antibodies to the Beta variant, which is more resistant to neutralization. Bamlanivimab and etesivimab lost their binding to the Beta variant, and only five of the antibodies bound to this variant (Extended Data Fig. 6). Thus, the escape of the Delta variant and other variants from neutralization is due to a reduction or loss of binding of the antibodies.

\section{Sensitivity of Delta to convalescent sera}

We examined the neutralization ability of sera from convalescent individuals. We first selected samples from 56 donors in a cohort of individuals from the French city of Orléans. All individuals had tested positive for SARS-CoV-2 infection by quantitative PCR with reverse transcription (qRT-PCR) or serology and included critical, severe, mild-to-moderate and asymptomatic cases of COVID-19 (Extended Data Table1). The individuals were not vaccinated at the time at which samples were taken. We have previously characterized the potency of these sera against D614G, Alpha and Beta isolates ${ }^{11}$. We analysed samples taken from individuals a median of 188 days after the onset of symptoms (referred to as month- 6 samples), and calculated the median half-maximal effective dilution $\left(\mathrm{ED}_{50}\right)$ for each combination of serum and virus (Extended Data Fig. 7a). With the Alpha variant, we obtained similar $\mathrm{ED}_{50}$ values in this series of experiments to those in our previous analysis ${ }^{11}$ (Extended Data Fig. 7b). We thus included our published data for D614G and Beta in the comparison. With the Delta variant, neutralization titres were significantly decreased by four- to sixfold when compared to the Alpha and D614G strains, respectively (Extended Data Fig. 7a). This reduction in neutralizing titres was similar against the Delta and Beta variants (Extended Data Fig. 7a).

We asked whether this neutralization profile was maintained for longer periods of time. We analysed sera from 47 individuals from another cohort consisting of healthcare workers from Strasbourg University Hospitals who had a qRT-PCR-confirmed diagnosis of COVID-19 and who experienced mild disease ${ }^{22,23}$. Twenty-six individuals were unvaccinated, and 21 had received a single dose of vaccine $7-81$ days before sampling. The samples were collected at a later time point (month 12), with a median of 330 and 359 days for unvaccinated and vaccinated individuals, respectively ${ }^{23}$ (Extended Data Table 1). As observed previously ${ }^{23}$, the neutralization activity was globally low at month 12 in unvaccinated individuals (Fig. 2a). There was a fourfold decrease in $\mathrm{ED}_{50}$ against the Beta and Delta variants relative to the Alpha variant (Fig. 2a). The 21 individuals within the month-12 cohort who had had a single dose of vaccine included 9 individuals who had received the AstraZeneca vaccine, 9 the Pfizer vaccine and 3 the Moderna vaccine. Sera from these vaccinated participants showed a marked increase in neutralizing antibody titres against the Alpha, Beta and Delta variants, as compared to convalescent individuals who had not been vaccinated (Fig. 2a). Therefore, as shown with other variants ${ }^{23,24}$, a single dose of vaccine boosts cross-neutralizing antibody responses to the Delta variant.

We then classified the individuals as neutralizers (individuals whose serum contained neutralizing antibodies that were detectable at the first serum dilution of $1 / 30$ ) and non-neutralizers, for the viral variants 
and the two cohorts (Extended Data Fig. 7c). Sera from between 76\% and $92 \%$ of individuals neutralized the four strains at month 6 . The fraction of neutralizers was lower in the second cohort at month 12, and this effect was particularly marked for the Beta and Delta variants. Sera from $88 \%$ of individuals neutralized the Alpha variant, whereas sera from only $47 \%$ of individuals neutralized the Delta variant. After vaccination, sera from $100 \%$ of the convalescent individuals neutralized the four strains (Extended Data Fig. 7c).

Thus, the Delta variant exhibits enhanced resistance to neutralization by sera from convalescent individuals who have not been vaccinated, particularly by one year after the infection.

\section{Sensitivity of Delta to sera from vaccinated individuals}

We next asked whether vaccine-elicited antibodies neutralized the Delta variant in individuals who had not previously been infected with SARS-CoV-2. We randomly selected 59 individuals from a cohort of vaccinated individuals in Orléans. The characteristics of these individuals are outlined in Extended Data Table 2. Sixteen individuals received the Pfizer vaccine. They were sampled at week 3 after the first dose and week 8 (corresponding to week 5 after the second dose). Thirteen individuals were also sampled at week 16 . Forty-three individuals received the AstraZeneca vaccine. Sera from 23 individuals were sampled after 1 dose (week 10) and from 20 other individuals after 2 doses (week 16, corresponding to week 4 after the second dose). We measured the potency of the sera against D614G and the Alpha, Beta and Delta variants (Fig. 2b, c).

With the Pfizer vaccine, after a single dose (at week 3 ) the levels of neutralizing antibodies were low against D614G, and almost undetectable against the Alpha, Beta and Delta variants (Fig. 2b). Titres significantly increased after the second dose. We observed a threefold and a sixteenfold reduction in the neutralization titres against the Delta and the Beta variants, respectively, when compared to the Alpha variant (Fig. 2b). Similar differences between strains were observed at a later time point (week 16), although titres were globally slightly lower (Extended Data Fig. 7b).

A similar pattern was observed with the AstraZeneca vaccine. A single dose (week 10) induced low levels of antibodies neutralizing the Delta and Beta variants, when compared to the D614G and Alpha strains (Fig. 2c). Four weeks after the second dose (week16), neutralizing titres were strongly increased. There was, however, a fivefold and a ninefold reduction in neutralization titres against the Delta and the Beta variants, respectively, relative to the Alpha variant (Fig. 2c).

We then classified the vaccine recipients as neutralizers and non-neutralizers, for the four viral strains (Extended Data Fig. 7d, e). For the Pfizer vaccine, sera from $13 \%$ of individuals neutralized the Delta variant after a single dose. Sera from 81 to $100 \%$ of individuals neutralized any of the four stains after the second dose, at week 8 . This fraction remained stable at week 16 , with the exception of the Beta variant, which was neutralized by sera from only $46 \%$ of the individuals. Sera from $74 \%$ and $61 \%$ of individuals that received a single dose of the AstraZeneca vaccine neutralized the D614G and the Alpha strains, respectively. This fraction sharply dropped with the Beta and the Delta variants, which were inhibited by only 4 and $9 \%$ of the sera, respectively. Four weeks after the second dose of the AstraZeneca vaccine, sera from $95-100 \%$ of individuals neutralized the four strains.

Therefore, a single dose of Pfizer or AstraZeneca either showed low or no efficiency against the Beta and the Delta variants. Both vaccines generated a neutralizing response that efficiently targeted the Delta variant only after the second dose.

\section{Discussion}

We studied the cross-reactivity of monoclonal antibodies to pre-existing SARS-CoV-2 strains, sera from convalescent individuals six or twelve months after the onset of COVID-19 symptoms and sera from recent recipients of a COVID-19 vaccine against an infectious Delta isolate. Some monoclonal antibodies, including bamlanivimab, lost their ability to bind to the spike protein and no longer neutralized the Delta variant. We also showed that the Delta variant is less sensitive to sera from naturally immunized individuals. Vaccination of convalescent individuals boosted the humoral immune response to well above the threshold of neutralization. These results strongly suggest that vaccination of previously infected individuals is likely to be protective against a large array of circulating viral strains, including the Delta variant.

In individuals who had not previously been infected with SARS-CoV-2, a single dose of either the Pfizer or the AstraZeneca vaccine induced a barely detectable level of neutralizing antibodies against the Delta variant. About $10 \%$ of the sera neutralized this variant. However, a two-dose regimen generated high sero-neutralization levels against the Alpha, Beta and Delta variants in individuals sampled at week 8 to week 16 after vaccination. Levels of neutralizing antibodies are highly predictive of immune protection from symptomatic SARS-CoV-2 infection $^{25}$. A recent report analysing all sequenced symptomatic cases of COVID-19 in England was used to estimate the effect of vaccination on infection ${ }^{26}$. Effectiveness was notably lower for the Delta variant than for the Alpha variant after one dose of the AstraZeneca or the Pfizer vaccine. The two-dose effectiveness against the Delta variant was estimated to be $60 \%$ and $88 \%$ for the AstraZeneca and the Pfizer vaccine, respectively ${ }^{26}$. Our neutralization experiments indicate that antibodies elicited by the Pfizer and AstraZeneca vaccines are efficacious against the Delta variant, but about three- to fivefold less potent than they are against the Alpha variant. There was no major difference in the levels of antibodies elicited by the Pfizer or the AstraZeneca vaccines.

Potential limitations of our work include the low number of vaccinated individuals analysed and the lack of characterization of cellular immunity, which may be more cross-reactive than the humoral response. Future work with more individuals and longer survey periods will help to characterize the role of humoral responses in the efficacy of vaccines against circulating variants.

Our results demonstrate that the emerging Delta variant partiallybut notably-escapes neutralizing monoclonal antibodies and polyclonal antibodies elicited by previous infection with SARS-CoV-2 or by vaccination.

\section{Online content}

Any methods, additional references, Nature Research reporting summaries, source data, extended data, supplementary information, acknowledgements, peer review information; details of author contributions and competing interests; and statements of data and code availability are available at https://doi.org/10.1038/s41586-021-03777-9. vaccinees. Clin. Infect. Dis. https://doi.org/10.1093/cid/ciab411 (2021).

Ferreira, I. et al. SARS-CoV-2 B.1.617 emergence and sensitivity to vaccine-elicited antibodies. Preprint at https://doi.org/10.1101/2021.05.08.443253 (2021).

3. Hoffmann, M. et al. SARS-CoV-2 variant B.1.617 is resistant to Bamlanivimab and evades antibodies induced by infection and vaccination. Cell Rep. https://doi.org/10.1016/ j.celrep.2021.109415 (2021)

4. Cherian, S. et al. Convergent evolution of SARS-CoV-2 spike mutations, L452R, E484Q and P681R, in the second wave of COVID-19 in Maharashtra, India. Preprint at https://doi. org/10.1101/2021.04.22.440932 (2021).

5. Edara, V.-V. et al. Infection and vaccine-induced neutralizing-antibody responses to the SARS-CoV-2 B.1.617 variants. New Eng. J. Med. https://doi.org/10.1056/NEJMc2107799 (2021).

6. Public Health England. Variants: distribution of case data, 11 June 2021. https://www.gov uk/government/publications/covid-19-variants-genomically-confirmed-case-numbers/ variants-distribution-of-case-data-11-june-2021 (2021).

7. Tada, T. et al. The spike proteins of SARS-CoV-2 B.1.617 and B.1.618 variants identified in India provide partial resistance to vaccine-elicited and therapeutic monoclonal antibodies. Preprint at https://doi.org/10.1101/2021.05.14.444076 (2021).

8. Liu, J. et al. BNT162b2-elicited neutralization of B.1.617 and other SARS-CoV-2 variants. Nature https://doi.org/10.1038/s41586-021-03693-y (2021). 


\section{Article}

9. Wall, E. C. et al. Neutralising antibody activity against SARS-CoV-2 VOCs B.1.617.2 and B.1.351 by BNT162b2 vaccination. Lancet 397, 2331-2333 (2021)

10. Buchrieser, J. et al. Syncytia formation by SARS-CoV-2-infected cells. EMBO J. 39 e106267 (2020).

11. Planas, D. et al. Sensitivity of infectious SARS-CoV-2 B.1.1.7 and B.1.351 variants to neutralizing antibodies. Nat. Med. 27, 917-924 (2021)

12. Rambaut, A. et al. A dynamic nomenclature proposal for SARS-CoV-2 lineages to assist genomic epidemiology. Nat. Microbiol. 5, 1403-1407 (2020).

13. Plante, J. A. et al. The variant gambit: COVID-19's next move. Cell Host Microbe 29, 508515 (2021)

14. Centers for Disease Control and Prevention. SARS-CoV-2 variant classifications and definitions. https://www.cdc.gov/coronavirus/2019-ncov/cases-updates/ variant-surveillance/variant-info.html (2021).

15. McCallum, M. et al. $\mathrm{N}$-terminal domain antigenic mapping reveals a site of vulnerability for SARS-CoV-2. Cell 184, 2332-2347 (2021).

16. Starr, T. N., Greaney, A. J., Dingens, A. S. \& Bloom, J. D. Complete map of SARS-CoV-2 RBD mutations that escape the monoclonal antibody LY-CoV555 and its cocktail with LY-CoV016. Cell Rep. Med. 2, 100255 (2021).

17. Barnes, C. O. et al. SARS-CoV-2 neutralizing antibody structures inform therapeutic strategies. Nature 588, 682-687 (2020).

18. Taylor, P. C. et al. Neutralizing monoclonal antibodies for treatment of COVID-19. Nat. Rev. Immunol. 21, 382-393 (2021).
19. Starr, T. N. et al. Prospective mapping of viral mutations that escape antibodies used to treat COVID-19. Science 371, 850-854 (2021).

20. Liu, L. et al. Potent neutralizing antibodies against multiple epitopes on SARS-CoV-2 spike. Nature 584, 450-456 (2020)

21. Wang, P. et al. Antibody resistance of SARS-CoV-2 variants B.1.351 and B.1.1.7. Nature $\mathbf{5 9 3}$ 130-135 (2021).

22. Fafi-Kremer, S. et al. Serologic responses to SARS-CoV-2 infection among hospital staff with mild disease in eastern France. EBioMedicine 59, 102915 (2020).

23. Gallais, F. et al. Anti-SARS-CoV-2 antibodies persist for up to 13 months and reduce risk of reinfection. Preprint at https://doi.org/10.1101/2021.05.07.21256823 (2021).

24. Krammer, F. et al. Antibody responses in seropositive persons after a single dose of SARS-CoV-2 mRNA vaccine. N. Engl. J. Med. 384, 1372-1374 (2021).

25. Khoury, D. S. et al. Neutralizing antibody levels are highly predictive of immune protection from symptomatic SARS-CoV-2 infection. Nat. Med. (2021).

26. Bernal, J. L. et al. Effectiveness of COVID-19 vaccines against the B.1.617.2 variant. Preprint at https://doi.org/10.1101/2021.05.22.21257658 (2021).

Publisher's note Springer Nature remains neutral with regard to jurisdictional claims in published maps and institutional affiliations.

(c) The Author(s), under exclusive licence to Springer Nature Limited 2021 


\section{Methods}

\section{Data reporting}

No statistical methods were used to predetermine sample size. The experiments were not randomized and the investigators were not blinded to allocation during experiments and outcome assessment. Our research complies with all relevant ethical regulations.

\section{Orléans cohort of convalescent and vaccinated individuals}

Since 27 August 2020, a prospective, monocentric, longitudinal, interventional cohort clinical study enrolling 170 individuals with SARS-CoV-2 infection (with different disease severities) and 59 healthy control individuals has been ongoing, and aims to describe the persistence of specific and neutralizing antibodies over a 24-month period. This study was approved by the Ile de France IV ethical committee. At enrolment, written informed consent was collected and participants completed a questionnaire that covered sociodemographic characteristics, virological findings (SARS-CoV-2 qRT-PCR results, including date of testing), clinical data (date of symptom onset, type of symptoms, hospitalization) and data related to anti-SARS-CoV-2 vaccination if ever (brand product, date of first and second doses). The serological status of participants was assessed every three months. Those who underwent anti-SARS-CoV-2 vaccination had regular blood sampling after the first dose of vaccine (ClinicalTrials.gov Identifier: NCT04750720). The primary outcome was the presence of antibodies to SARS-CoV-2 spike protein as measured with the S-Flow assay. The secondary outcome was the presence of neutralizing antibodies as measured with the S-Fuse assay. For the present study, we selected 56 convalescent individuals and 59 vaccinated individuals ( 16 with Pfizer and 43 with AstraZeneca). Study participants did not receive any compensation.

\section{Strasbourg cohort of convalescent individuals}

Since April 2020, a prospective, monocentric, longitudinal, interventional cohort clinical study enrolling 308 hospital staff from the Strasbourg University Hospitals who had a qRT-PCR-confirmed diagnosis of SARS-CoV-2 infection has been ongoing (ClinicalTrials.gov Identifier: NCT04441684). At enrolment (from 17 April 2020), written informed consent was collected and participants completed a questionnaire that covered sociodemographic characteristics, virological findings (SARS-CoV-2 qRT-PCR results, including date of testing) and clinical data (date of symptom onset, type of symptoms, hospitalization). This study was approved by the Institutional Review Board of Strasbourg University Hospital. The serological status of the participants has been described at months 3 and 6 after the onset of symptoms $^{22,23}$. Laboratory identification of SARS-CoV-2 was performed at least 10 days before inclusion by qRT-PCR testing on nasopharyngeal swab specimens according to current guidelines (Institut Pasteur; WHO technical guidance). The assay targets two regions of the viral RNA-dependent RNA polymerase (RdRp) gene with a threshold of detection of 10 copies per reaction. The primary outcome was the presence of antibodies to the SARS-CoV-2 spike protein as measured with the S-Flow assay. The secondary outcome was the presence of neutralizing antibodies as measured with the S-Fuse assay. For the present study, we randomly selected 47 convalescent individuals at month 12 (26 unvaccinated and 21 vaccinated). Study participants did not receive any compensation.

\section{Phylogenetic analysis}

All SARS-CoV-2 sequences available on the GISAID EpiCov database as of 21 May 2021 were retrieved. A subset of complete and high-coverage sequences, as indicated in GISAID, assigned to lineages B.1.617.1, B.1.617.2 and B.1.617.3 were randomly subsampled to contain up to five sequences per country and epidemiological week in $\mathrm{R}$ with the packages tidyverse and lubridate. Together with a single B.1.617 sequence this subset was included in the global SARS-CoV-2 phylogeny reconstructed with augur and visualized with auspice as implemented in the Nextstrain pipeline (https://github.com/nextstrain/ncov; version from 21 May $2021)^{27}$. Within Nextstrain, a random subsampling approach capping a maximum number of sequences per global region was used for the contextual non-B.1.617 sequences. Acknowledgement of the contributing and originating laboratories for all sequences used in the analysis is provided in Supplementary Table 1.

\section{Three-dimensional mapping of mutations on B1.617.2 and other variants to the surface of the spike protein}

Panels in Extended Data Fig. 4 were prepared with the PyMOL Molecular Graphics System, v.2.1 (Schrödinger). The atomic model used (Protein Data Bank code: 6 XR8) has been previously described ${ }^{28}$.

\section{S-Fuse neutralization assay}

U2OS-ACE2 GFP1-10 or GFP 11 cells, also termed S-Fuse cells, become $\mathrm{GFP}^{+}$when they are productively infected by SARS-CoV-2 ${ }^{10,11}$. Cells tested negative for mycoplasma. Cells were mixed (ratio 1:1) and plated at $8 \times 10^{3}$ per well in a $\mu$ Clear 96-well plate (Greiner Bio-One). The indicated SARS-CoV-2 strains were incubated with serially diluted monoclonal antibodies or sera for $15 \mathrm{~min}$ at room temperature and added to S-Fuse cells. The sera were heat-inactivated $30 \mathrm{~min}$ at $56^{\circ} \mathrm{C}$ before use. Eighteen hours later, cells were fixed with $2 \%$ paraformaldehyde (PFA), washed and stained with Hoechst (dilution 1:1,000, Invitrogen). Images were acquired with an Opera Phenix high-content confocal microscope (PerkinElmer). The GFP area and the number of nuclei were quantified using Harmony software (PerkinElmer). The percentage of neutralization was calculated using the number of syncytia as value with the following formula: $100 \times(1-$ (value with serum - value in 'non-infected')/(value in 'no serum' - value in 'non-infected')). The neutralizing activity of each serum was expressed as the $\mathrm{ED}_{50}$ value. $\mathrm{ED}_{50}$ values (in $\mu \mathrm{g} \mathrm{ml}^{-1}$ for monoclonal antibodies and in dilution values for sera) were calculated with a reconstructed curve using the percentage of the neutralization at the different concentrations.

\section{Clinical history of the patient infected with B.1.617.2}

A 54-year-old man was admitted on 27 April 2021 to the emergency department of the Hôpital Européen Georges Pompidou hospital in Paris, France, for an acute respiratory distress syndrome with fever. He had no medical background and came back from a trip to India (West Bengal and a few days spent in Delhi) 10 days before (17 April 2021), where he stayed 15 days for his work. The onset of symptoms (abdominal pain and fever) was approximately 18 April 2021 . The nasopharyngeal swab tested positive for SARS-CoV-2 at his date of admission. Lung tomo-densitometry showed a mild (10-25\%) COVID-19 pneumonia without pulmonary embolism. He initially received oxygen therapy $\left(21 \mathrm{~min}^{-1}\right)$, dexamethasone (6 mg per day) and enoxaparin $(0.4 \mathrm{ml}$ twice a day). His respiratory state worsened on day 3 (30 April 2021). He was transferred to an intensive care unit, in which he received high-flow oxygen therapy (maximum $12 \mathrm{I} \mathrm{min}^{-1}$ ). His respiratory condition improved, and he was transferred back to a conventional unit on day 8 (5 May 2021). He was discharged from hospital on day 15 (May 102021$).$

\section{Virus strains}

The reference D614G strain (hCoV-19/France/GE1973/2020) was supplied by the National Reference Centre for Respiratory Viruses hosted by the Institut Pasteur and headed by S. van der Werf. This viral strain was supplied through the European Virus Archive-Global (EVAg) platform, a project that has received funding from the European Union's Horizon 2020 research and innovation program under grant agreement no. 653316. The variant strains were isolated from nasal swabs using Vero E6 cells and amplified by one or two passages. B.1.1.7 originated from an individual in Tours (France) who had returned from the UK. B.1.351 (hCoV-19/France/IDF-IPP00078/2021) originated from an individual in 
Creteil (France). B.1.617.2 was isolated from a nasopharyngeal swab of a hospitalized patient who had returned from India, as described above. The swab was provided and sequenced by the Laboratoire de Virologie of the Hopital Européen Georges Pompidou (Assistance Publique des Hôpitaux de Paris). All individuals provided informed consent for the use of the biological materials. Titration of viral stocks was performed on Vero E6, with a limiting dilution technique allowing a calculation of TCID50, or on S-Fuse cells. Viruses were sequenced directly on nasal swabs, and after one or two passages on Vero cells. Sequences were deposited in the GISAID database immediately after their generation, with the following IDs: D614G: EPI_ISL_414631; B.1.1.7: EPI_ISL_735391; B.1.1.351:EPI_ISL_964916; B.1.617.2: ID: EPI_ISL_2029113.

\section{Flow cytometry}

Vero cells were infected with the indicated viral strains at a multiplicity of infection (MOI) of 0.1. Two days after, cells were detached using PBS-EDTA and transferred into U-bottom 96 -well plates $(50,000$ cells per well). Cells were fixed in 4\% PFA for $15-30 \mathrm{~min}$ at room temperature. Cells were then incubated for 15-30 min at room temperature with the indicated monoclonal antibodies $\left(1 \mu \mathrm{g} \mathrm{ml}^{-1}\right)$ in PBS, $1 \%$ BSA, $0.05 \%$ sodium azide and $0.05 \%$ saponin. Cells were washed with PBS and stained using anti-IgG AF647 (1:600 dilution) (Thermo Fisher Scientific). Stainings were also performed on control uninfected cells. Data were acquired on an Attune Nxt instrument using Attune Nxt Software v.3.2.1 (Life Technologies) and analysed with FlowJo v.10.7.1 (Becton Dickinson).

\section{Antibodies}

The four therapeutic antibodies were provided by CHR Orleans. Human anti-SARS-CoV2 monoclonal antibodies were cloned from S-specific blood memory B cells of individuals who were convalescing from COVID-19 (C.P. et al., manuscript in preparation). Recombinant human IgG1 monoclonal antibodies were produced by co-transfection of Freestyle 293-F suspension cells (Thermo Fisher Scientific) as previously described ${ }^{29}$, purified by affinity chromatography using protein $\mathrm{G}$ sepharose 4 fast flow beads (GE Healthcare) and validated by enzyme-linked immunosorbent assay (ELISA) against the trimeric S, RBD, S2 and NTD proteins (C.P. et al., manuscript in preparation).

\section{Statistical analysis}

Flow cytometry data were analysed with FlowJo v.10 software (TriStar). Calculations were performed using Excel 365 (Microsoft). Figures were drawn using GraphPad Prism 9. Statistical analysis was conducted using GraphPad Prism 9. Statistical significance between different groups was calculated using the tests indicated in each figure legend.

\section{Reporting summary}

Further information on research design is available in the Nature Research Reporting Summary linked to this paper.

\section{Data availability}

All data supporting the findings of this study are available within the Article or from the corresponding authors upon request. Viral sequences are available upon request and were deposited at GISAID (https://www.gisaid.org/) under the following numbers: hCoV-19/ France/GE1973/2020 (D614G): EPI_ISL_414631; Alpha (B.1.1.7): EPI_ ISL_735391; Beta (B.1.351): EPI_ISL_964916 and Delta (B.1.617.2): EPI_ ISL_2029113. Source data are provided with this paper.

27. Hadfield, J. et al. Nextstrain: real-time tracking of pathogen evolution. Bioinformatics $\mathbf{3 4}$ 4121-4123 (2018).

28. Cai, Y. et al. Distinct conformational states of SARS-CoV-2 spike protein. Science 369, 1586-1592 (2020).

29. Lorin, V. \& Mouquet, H. Efficient generation of human IgA monoclonal antibodies. J. Immunol. Methods 422, 102-110 (2015).

30. Tzou, P. L. et al. Coronavirus antiviral research database (CoV-RDB): an online database designed to facilitate comparisons between candidate anti-coronavirus compounds. Viruses 12, 1006 (2020).

Acknowledgements We thank N. Casartelli for critical reading of the manuscript; P. Guardado Calvo for discussion; the individuals who participated in the study; members of the Virus and Immunity Unit for discussions and help; N. Aulner and the UTechS Photonic Biolmaging (UPBI) core facility (Institut Pasteur), a member of the France Biolmaging network, for image acquisition and analysis; and the $\mathrm{DRCl}, \mathrm{ClC}$, Médecine du Travail and Pôle de Biologie teams (CHU de Strasbourg) for the management of the Strasbourg cohort and serology testing. The Opera system was co-funded by the Institut Pasteur and the Région île de France (DIM1Health). Work in the O.S. laboratory is funded by the Institut Pasteur, Urgence COVID-19 Fundraising Campaign of Institut Pasteur, Fondation pour la Recherche Médicale (FRM), ANRS, the Vaccine Research Institute (ANR-10-LABX-77), Labex IBEID (ANR-10-LABX-62-IBEID), ANR/FRM Flash Covid PROTEO-SARS-CoV-2 and IDISCOVR. Work in UPBI is funded by grant ANR-

10-INSB-04-01 and the Région Île-de-France program DIM1-Health. D.P. is supported by the Vaccine Research Institute. The H.M. laboratory is funded by the Institut Pasteur, the Milieu Intérieur Program (ANR-10-LABX-69-01), INSERM, REACTing, EU (RECOVER) and Fondation de France (00106077) grants. The S.F.K. laboratory is funded by Strasbourg University Hospitals (SeroCoV-HUS; PRI 7782), the Programme Hospitalier de Recherche Clinique (PHRC N 2017HUS no. 6997), the Agence Nationale de la Recherche (ANR-18-CE17-0028), Laboratoire d'Excellence TRANSPLANTEX (ANR-11-LABX-0070_TRANSPLANTEX) and the Institut National de la Santé et de la Recherche Médicale (UMR_S 1109). The E.S.-L. laboratory is funded by the Institut Pasteur and the French Government's Investissement d'Avenir programme, Laboratoire d'Excellence 'Integrative Biology of Emerging Infectious Diseases' (grant no. ANR-10-LABX62-IBEID). The funders of this study had no role in study design, data collection, analysis and interpretation, or writing of the Article.

Author contributions Experimental strategy design, experiments: D.P., D.V., A.B., I.S., F.G.-B., M.M.R., F.P., T.B., E.S.-L. and F.A.R. Vital materials: D.V., C.P., N.R., J.P., M.P., F.G., P.G., A.V., J.L.G., L.C., N.K.-C., D.E., L.B., A.S., H.P., L.H., S.F.-K., T.P. and H.M. Manuscript writing: D.P., T.B., E.S.-L., F.A.R. and O.S. Manuscript editing: D.V., M.M.R., H.P., L.H., S.F.K., T.P. and H.M.

Competing interests C.P., H.M., O.S, T.B. and F.A.R. have a pending patent application for the anti-RBD monoclonal antibodies described in the present study (PCT/FR2021/070522). F.A.R. is a member of the Board of MELETIOS Therapeutics and of the Scientific Advisory Board of eureKARE. The remaining authors declare no competing interests.

\section{Additional information}

Supplementary information The online version contains supplementary material available at https://doi.org/10.1038/s41586-021-03777-9.

Correspondence and requests for materials should be addressed to T.B. or O.S.

Peer review information Nature thanks the anonymous reviewers for their contribution to the peer review of this work.

Reprints and permissions information is available at http://www.nature.com/reprints. 
a

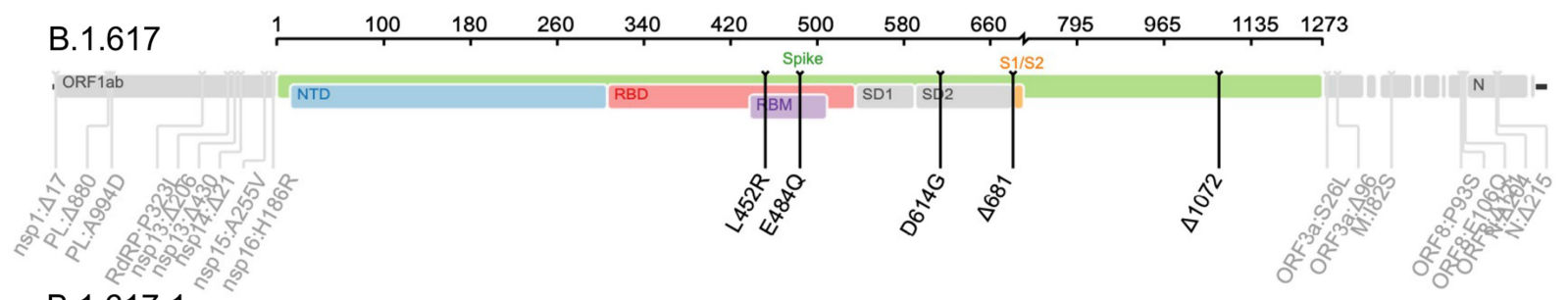

\section{B.1.617.1}

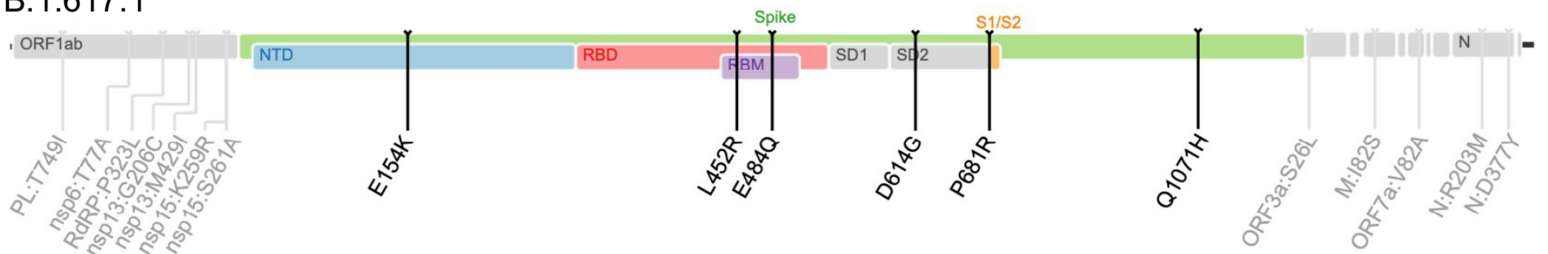

B.1.617.2 (Delta), EPI_ISL_2029113
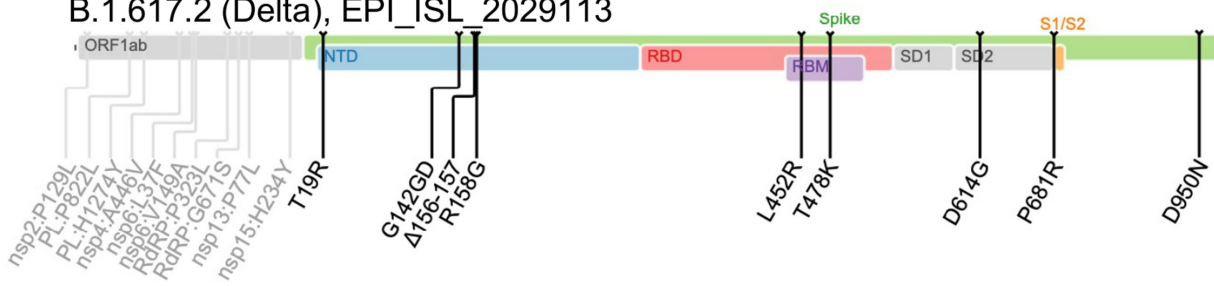

\section{B.1.617.3}
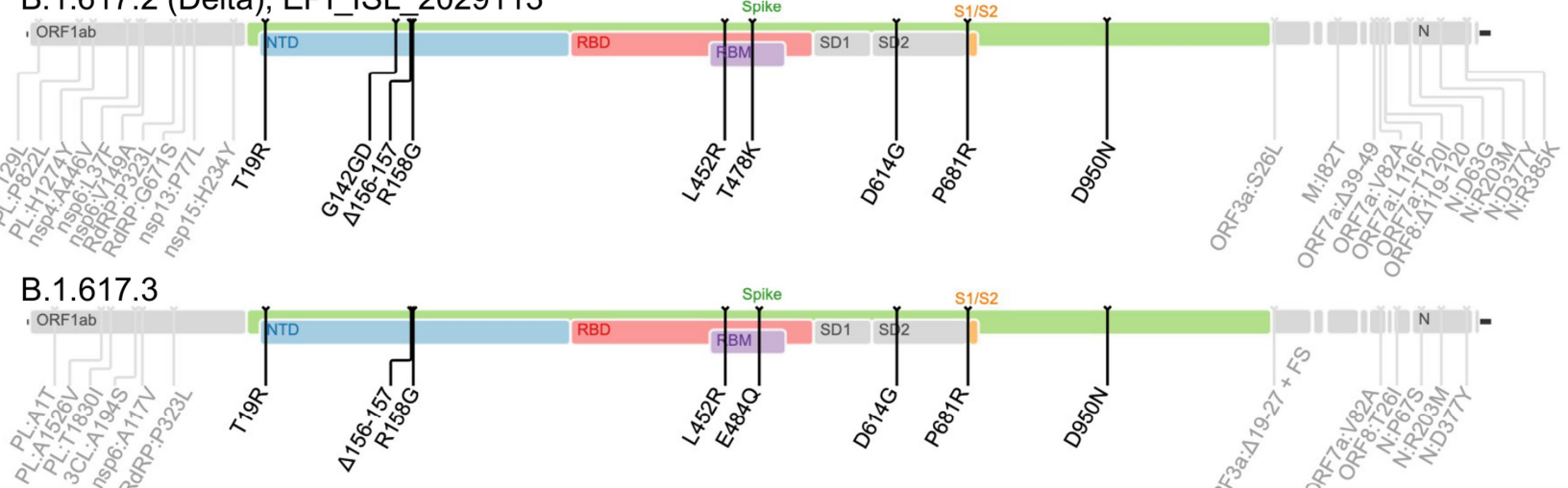

vivivis

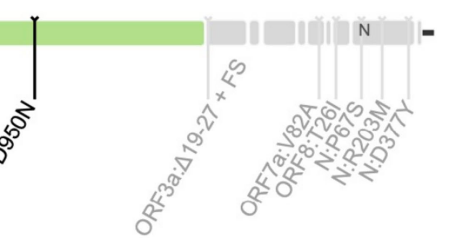

b

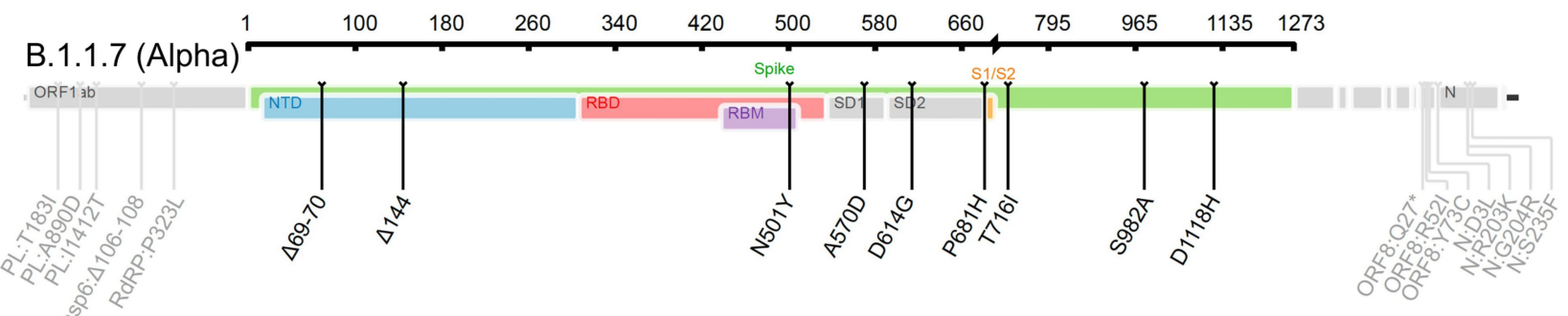

P.1 (Gamma)

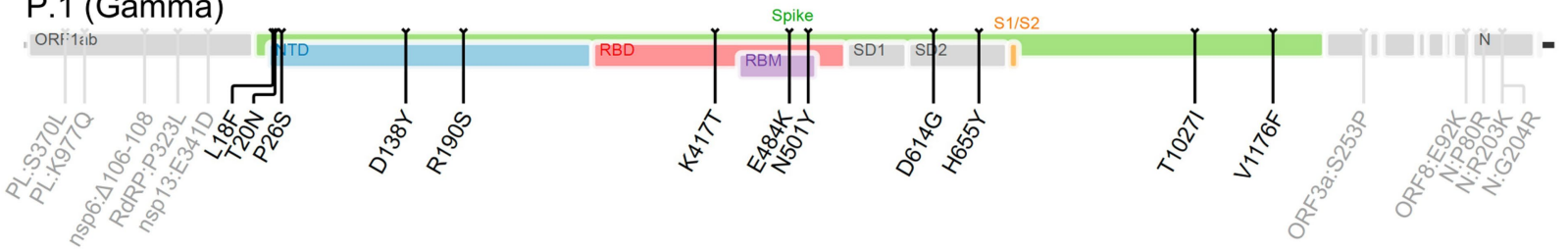

B.1.351 (Beta)

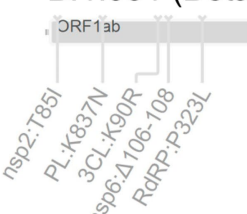

Extended Data Fig. 1 | Schematic overview of the B.1.617 sublineage and VOCs. a, b, Schematic overview of the B.1.617 sublineage (a) and the VOCs

B.1.1.7 (Alpha), P1 (Gamma) and B.1.351 (Beta) (b). Consensus sequences with a focus on the spike protein were built with the Sierra tool ${ }^{30}$. Amino acid modifications in comparison to the ancestral Wuhan-Hu-1 sequence (NC_045512) are indicated. 


\section{Article}
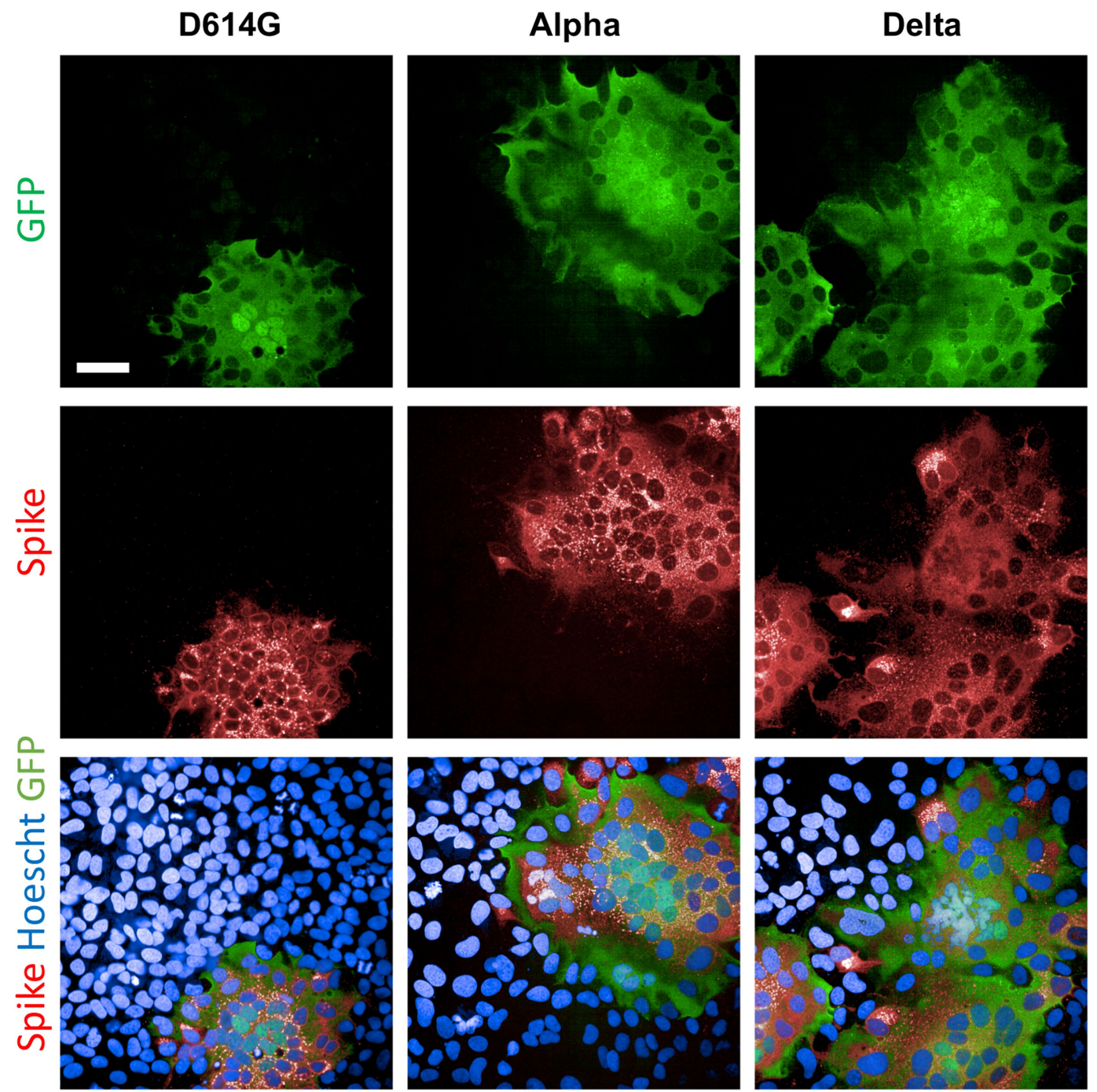

Extended Data Fig. 2 |SARS-CoV-2 variants induce syncytia in S-Fuse cells. S-Fuse cells were exposed to the indicated SARS-CoV-2 strain (at a MOI of $10^{-3}$ ). The cells become $\mathrm{GFP}^{+}$when they fuse together. After $20 \mathrm{~h}$, infected cells were stained with anti-spike antibodies and Hoechst to visualize nuclei. Syncytia (green), spike protein (red) and nuclei (blue) are shown. Representative images from three independent experiments are shown. Scale bar, $50 \mu \mathrm{m}$. 


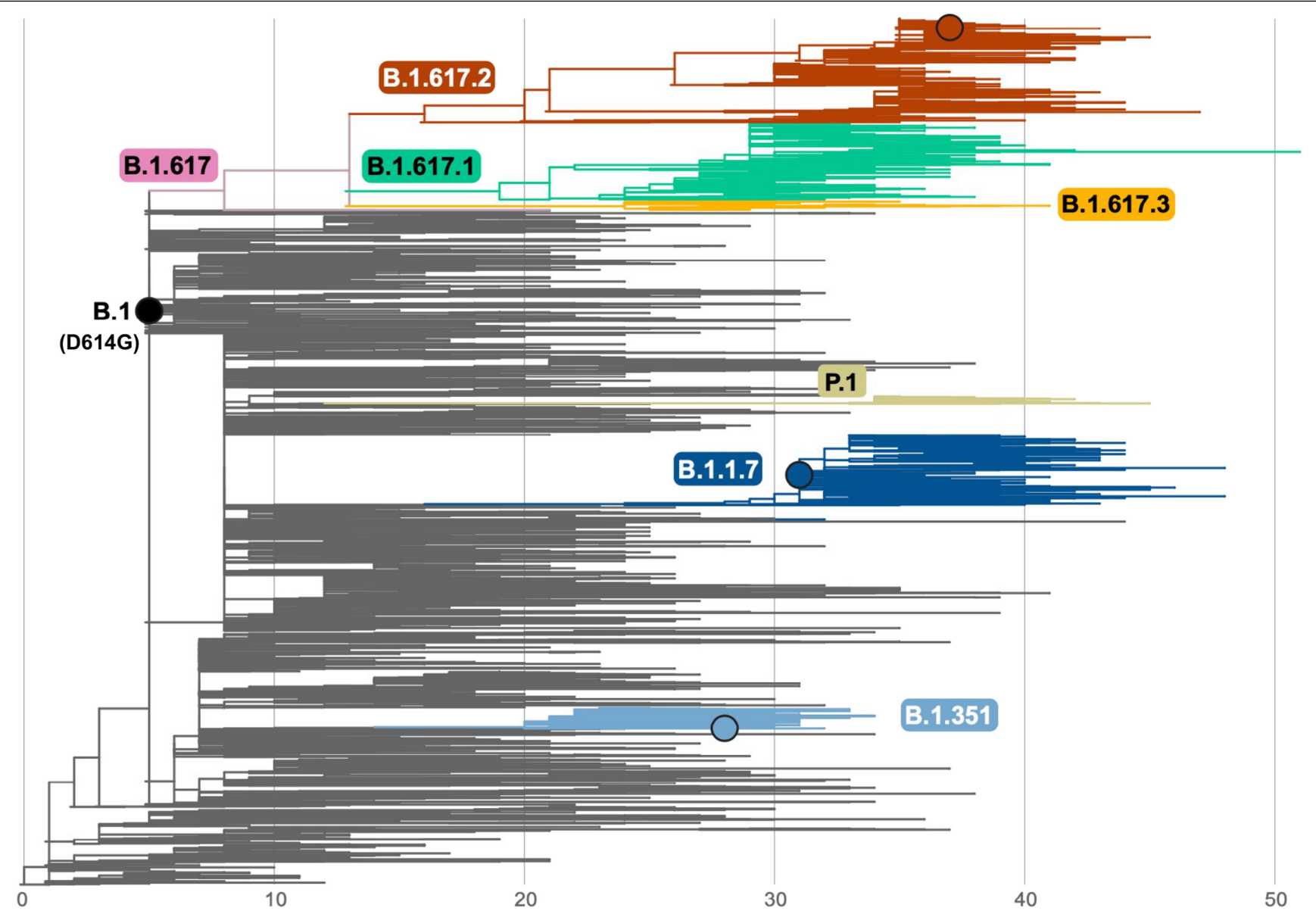

Extended Data Fig. 3 | Global phylogeny of SARS-CoV-2 highlighting the B.1.617 lineage. The maximum likelihood tree was inferred using IQ-Tree, as implemented in the Nextstrain pipeline on a subsampled dataset of 3,794 complete genomes. Branch lengths are scaled according to the number of nucleotide substitutions from the root of the tree. The branches corresponding to key lineages are coloured: B.1.1.7, dark blue; B.1.351, light blue; P.1, beige; B.1.617, pink; B.1.617.1, green; B.1.617.2, red; and B.1.617.3, orange. A black circle indicates the position of the viruses studied here. 
a b

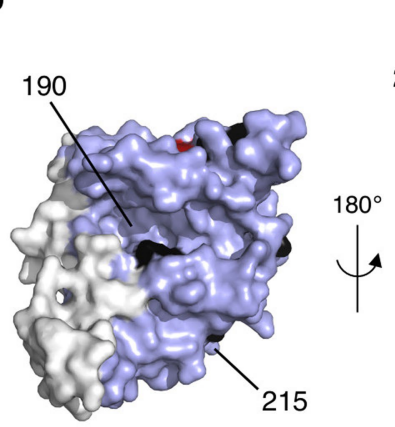

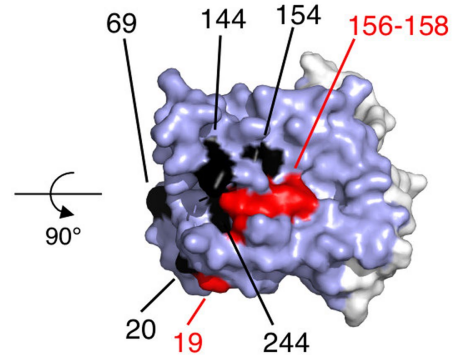

26

$19^{138}$

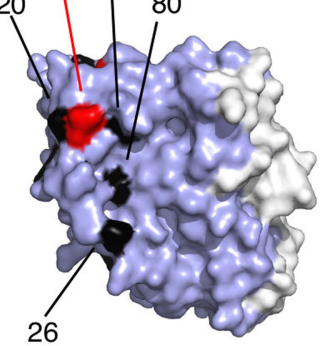

58

C

Removal of

protomer A
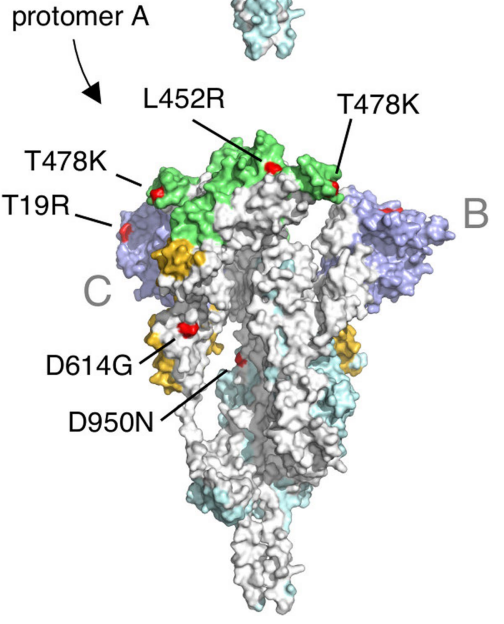

C

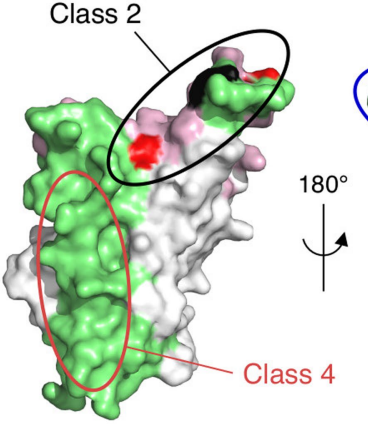

EXPOSED

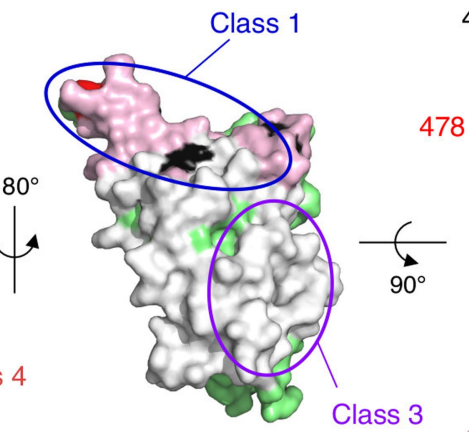

BURIED

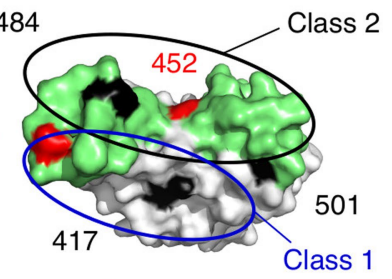

Class 1

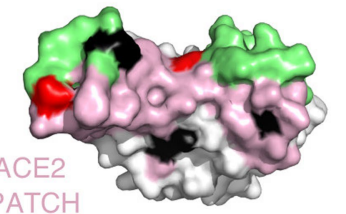

Extended Data Fig. 4 | Mapping mutations of the Delta variant and other variants of concern to the surface of the spike protein. $a$, The spike protein trimer (Protein Data Bank code: 6XR8, corresponding to a closed spike trimer with all three RBDs in the 'down' conformation) is shown with its surface coloured according to domains: NTD in dark blue, RBD in green, the remainder of $\mathrm{S} 1$ in yellow and $\mathrm{S} 2$ in light blue. Interfaces between protomers were left white to help visualize the boundaries of the protomers. The three polypeptide chains in the trimer were arbitrarily defined as A, B and C. Surface patches corresponding to residues mutated in the Delta variant are coloured in red. The bottom panel has the front protomer (chain $\mathrm{A}$ ) removed to show the trimer interface (buried regions in the trimer are in white). The mutations in Delta are labelled in the bottom panel.b, NTD shown in three orthogonal views. The left panel corresponds roughly to the orientation seen in chain $B$ in $\mathbf{a}$, and the middle panel shows a view from the back. The right panel shows a view from the top of the trimer. Mutations found in the main variants of concern are indicated. The mutations found in the Delta variant are in red.c, RBD shown in three orthogonal views, coloured according to solvent exposure in the context of the closed spike: green and white indicate exposed and buried surfaces, as in a. The ACE2-binding surface is coloured in pink. The left panel shows a view from the top of the trimer, and the middle panel a view from below. The right panels show a view down the ACE2-binding surface, highlighted in pink in the bottom panel. Mutations found in the main variants of concern are indicated. The mutations found in the Delta variant are in red. The ovals indicate the epitope regions of the four main classes of anti-RBD neutralizing antibodies. Note that the mutations on the RBD cluster all around the ACE2 patch. Panels were prepared with the PyMOL Molecular Graphics System, v.2.1 (Schrödinger). 

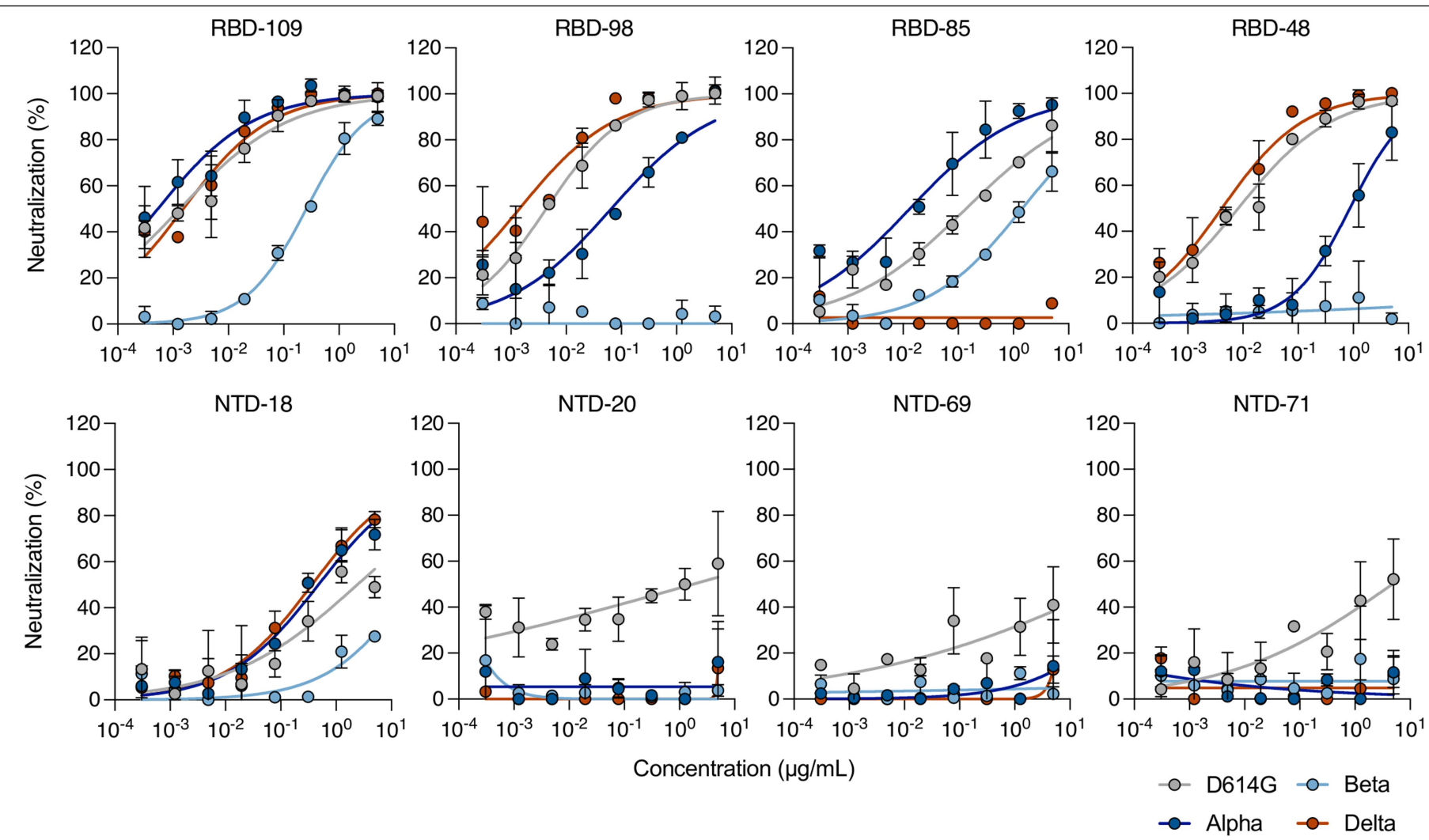

Extended Data Fig. 5 | Neutralization of the SARS-CoV-2 variants D614G, Alpha, Beta and Delta by monoclonal antibodies that target the RBD and the NTD domains. Neutralization curves of monoclonal antibodies. Doseresponse analysis of the neutralization by four anti-RBD and four anti-NTD antibodies on the D614G strain (grey) and the Alpha (dark blue), Beta (light blue) and Delta (orange) variants. Data are mean \pm s.d. of three independent experiments. 
a

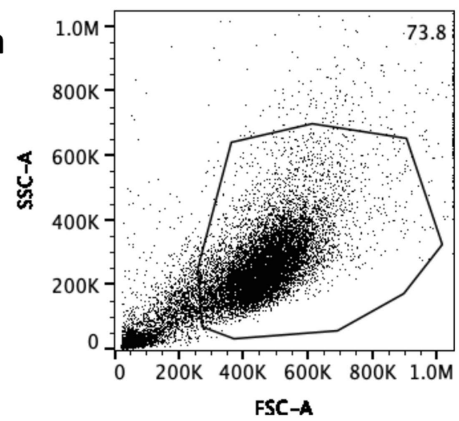

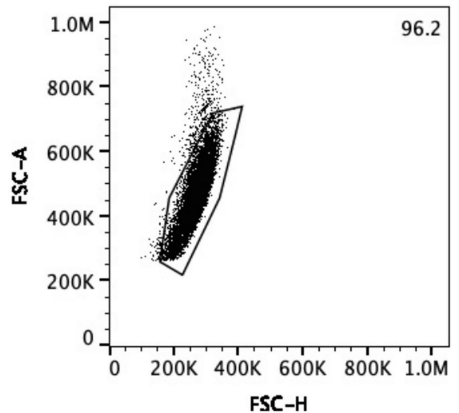

FSC-H b

Bamlanivimab

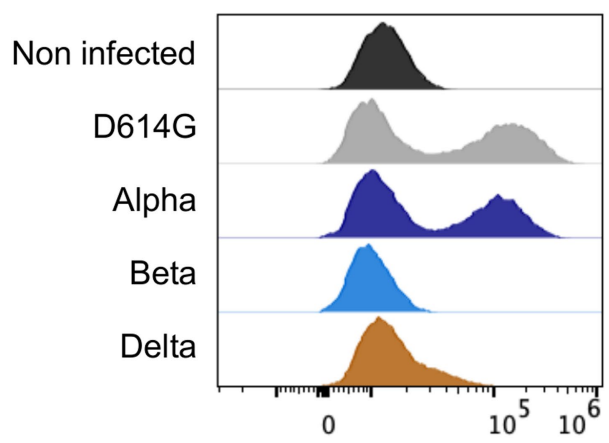

Imdevimab

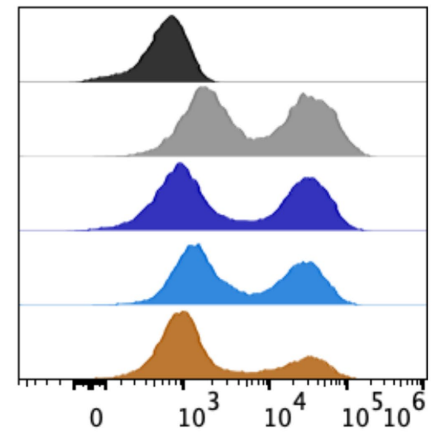

RBD-85

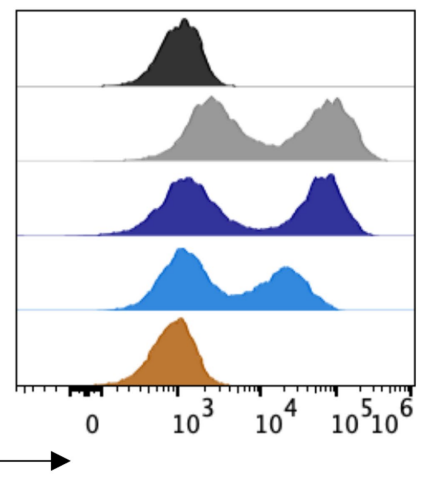

Anti-Human IgG - AlexaFluor647

C

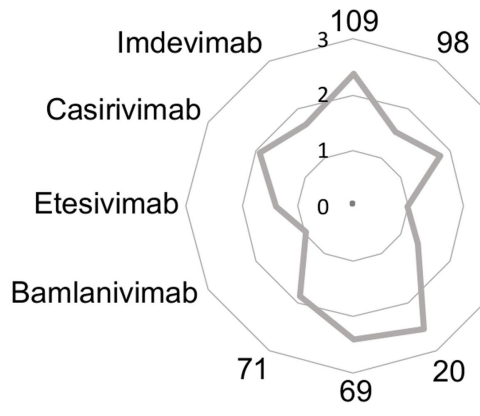

Beta

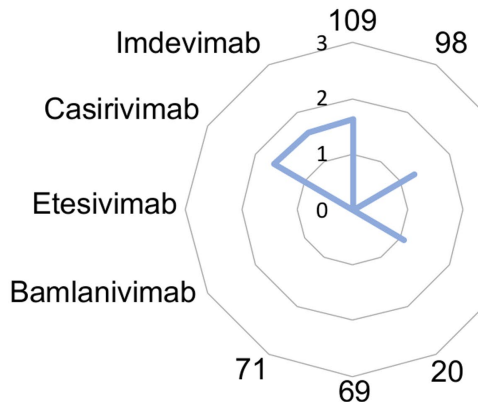

Extended Data Fig. 6 | Binding of anti-SARS-CoV-2 monoclonal antibodies to Vero cells infected with the SARS-CoV-2 variants D614G, Alpha, Beta and Delta. Vero cells were infected with the indicated variants at a MOI of 0.1. After $48 \mathrm{~h}$, cells were stained with anti-SARS-CoV-2 monoclonal antibodies $\left(1 \mu \mathrm{g} \mathrm{ml}^{-1}\right)$ and analysed by flow-cytometry. a, Gating strategy.b, Histograms show the

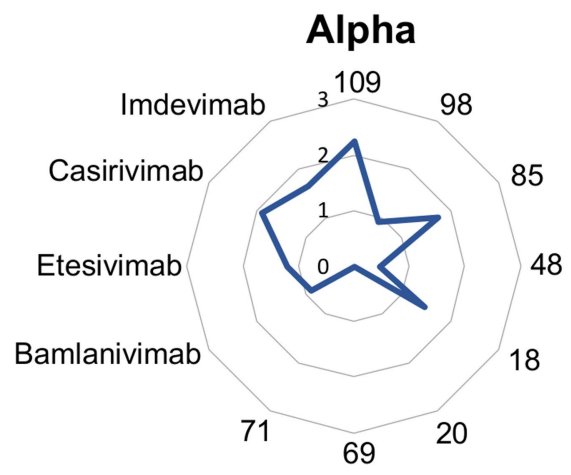

Delta

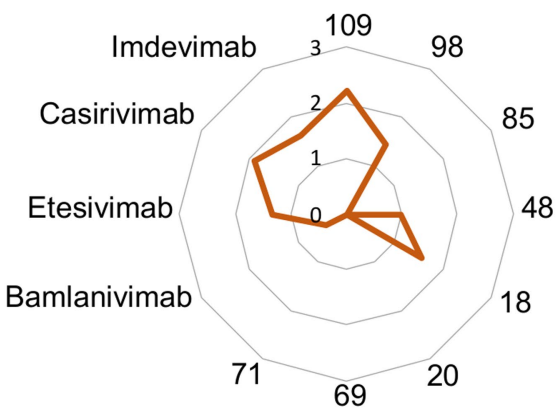

binding of bamlanivimab, imdevimab and RBD-85 to Vero cells infected with the indicated variants. $c$, Radar charts represent for each antibody the logarithm of the mean of fluorescent intensity of the staining, relative to the non-infected condition. Data are representative of three independent experiments. 
a

Orléans Cohort

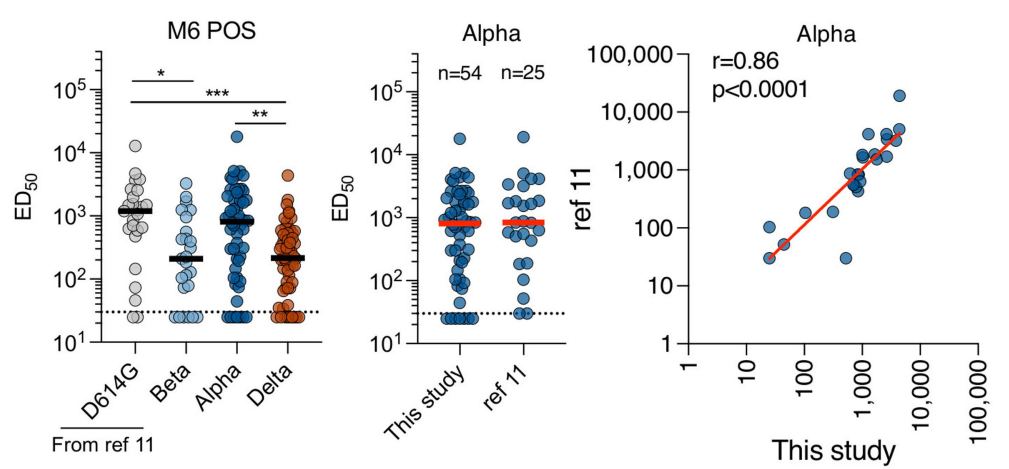

b

Pfizer

W16 after vaccination (W13 after $2^{\text {nd }}$ dose)

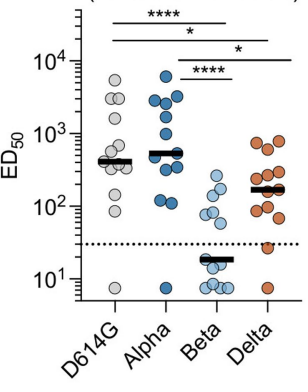

c Orléans Cohort

Strasbourg Cohort
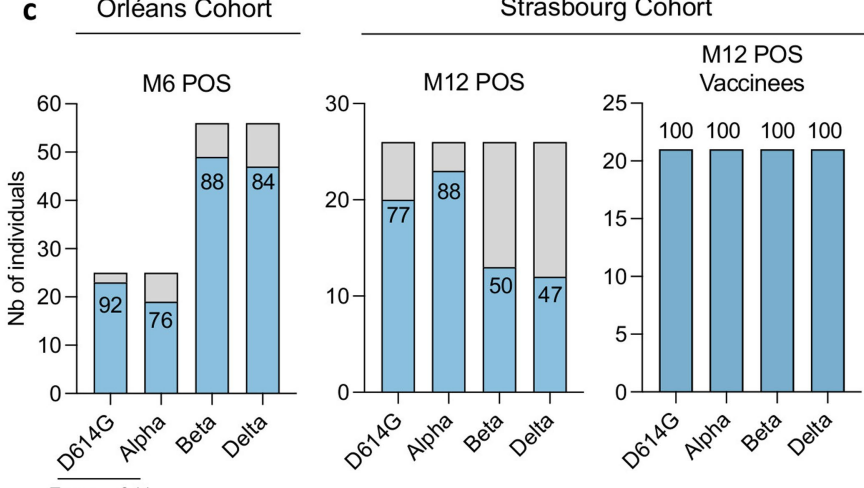

d

Pfizer
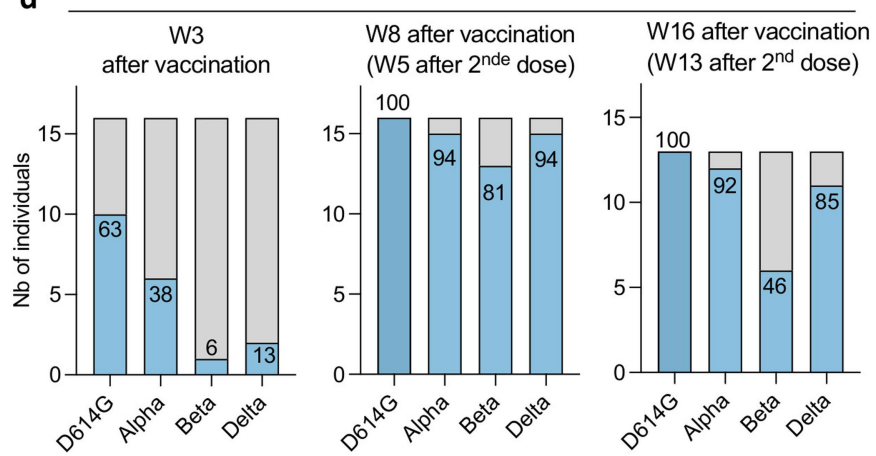

e

AstraZeneca
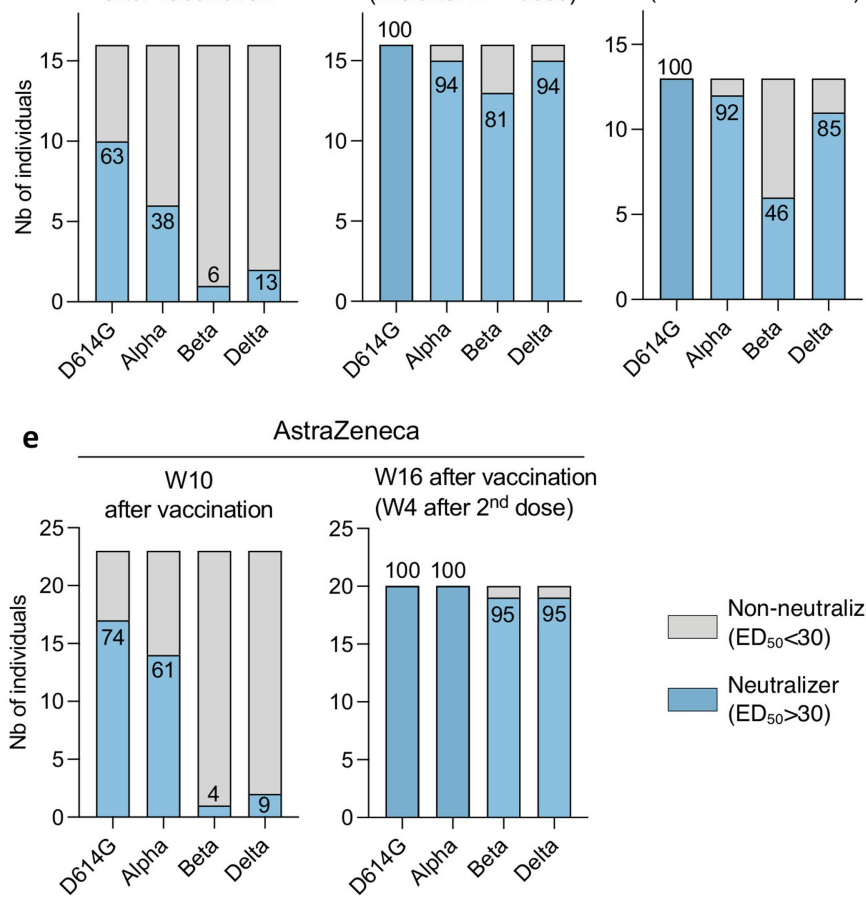

Non-neutralizer

$\left(\mathrm{ED}_{50}<30\right)$

Neutralizer

$\left(E D_{50}>30\right)$

Extended Data Fig. 7 |See next page for caption. 


\section{Article}

Extended Data Fig. 7 | Sensitivity of the SARS-CoV-2 variants D614G, Alpha, Beta and Delta to sera from convalescent individuals and vaccinated

individuals. $\mathbf{a}, \mathrm{ED}_{50}$ values for the neutralizing activity of sera from convalescent individuals from the Orléans cohort against the four viral variants. Samples were collected 6 months after the onset of symptoms (M6 POS). The sensitivity of the D614G and Alpha variants to sera from 25 individuals has been described previously ${ }^{11}$. Fifty-six sera (including the 25 previous sera) were tested against the Beta and Delta variants. Neutralization data obtained in this study and in ref. ${ }^{11}$ were compared (middle) and correlated (right). Similar results were obtained, allowing the datasets to be bridged. Data are mean from two independent experiments. The dotted line indicates the limit of detection $\left(\mathrm{ED}_{50}=30\right)$. A two-sided Kruskal-Wallis test with Dunn's multiple comparison was performed between each of the viral strains. ${ }^{*} P<0.05,{ }^{* *} P<0.01,{ }^{* * *} P<0.001,{ }^{* * * *} P<0.0001$. D614G versus Beta, $P=0.0153$; D614G versus Delta, $P=0.0008$; Alpha versus Delta, $P=0.0014$. b, $\mathrm{ED}_{50}$ values for the neutralizing activity of sera from individuals who received a Pfizer vaccine, sampled at week 16 (corresponding to week 13 after the second dose).
Data are mean from two independent experiments. The dotted line indicates the limit of detection $\left(\mathrm{ED}_{50}=30\right)$. A two-sided Kruskal-Wallis test with Dunn's multiple comparison was performed between each viral strain. ${ }^{*} P<0.05$, ${ }^{* *} P<0.01,{ }^{* * *} P<0.001,{ }^{* * * *} P<0.0001$. D614G versus Beta, $P<0.0001$; D614G versus Delta, $P=0.0375$; Alpha versus Beta, $P<0.0001$; Alpha versus Delta, $P=0.0375$. c-e, Fraction of neutralizers in the cohorts of convalescent or vaccinated individuals. Individuals with an $\mathrm{ED}_{50}$ of neutralization above 30 were categorized as neutralizers and are indicated in blue. Non-neutralizers are in grey. c, Analysis of convalescent individuals from the Orléans cohort collected at month 6 (left; related to a), and unvaccinated (middle; related to Fig. 2a) or vaccinated (right, related to Fig. 2a) individuals from the Strasbourg cohort collected at month 12.d, Sera from recipients of the Pfizer vaccine were sampled at week 3 (left), week 8 (middle) (both related to Fig. 2c) and week 16 (right; related to $b$ ) after vaccination. $\mathbf{e}$, Sera from recipients of the AstraZeneca vaccine were sampled at week 10 (left) and week 16 (right) after vaccination (related to Fig. 2c). The numbers indicate the percentage of neutralizers. 
Extended Data Table 1 | Characteristics of the two cohorts of convalescent individuals

a.

Orléans Cohort: M6 POS

$n=56$

\begin{tabular}{lll}
\hline Sex & Female & 29 \\
& Male & 27 \\
\hline Age (Median; range) & & $53(22 ; 77)$ \\
\hline Severity & Critical & 13 \\
& Severe & 15 \\
& Mild-Moderate & 16 \\
& Asymptomatic & 12 \\
\hline HIV & & 3 \\
\hline PCR & & 53 \\
\hline Anti-S (S-Flow) & & 56 \\
\hline Sampling days POS (median; range) & & $188(114 ; 205)$ \\
\hline
\end{tabular}

b.

Convalescent Vaccinated

Convalescent

Strasbourg Cohort : M12 POS

$n=26$

$\mathrm{n}=\mathbf{2 1}$

\begin{tabular}{|c|c|c|c|}
\hline \multicolumn{4}{|l|}{ Sex } \\
\hline & Female & 21 & 18 \\
\hline & Male & 5 & 3 \\
\hline $\begin{array}{l}\text { Age (Median; } \\
\text { range) }\end{array}$ & & $36(24 ; 60)$ & $44(23 ; 62)$ \\
\hline \multicolumn{4}{|l|}{ Severity } \\
\hline & Critical & 0 & 0 \\
\hline & Severe & 0 & 0 \\
\hline & Mild-Moderate & 26 & 21 \\
\hline & Asymptomatic & 0 & 0 \\
\hline PCR & & 26 & 21 \\
\hline Anti-S (Abott) & & 26 & 21 \\
\hline \multicolumn{4}{|l|}{ Vaccine } \\
\hline & AstraZeneca & 0 & 9 \\
\hline & Pfizer & 0 & 9 \\
\hline & Moderna & 0 & 3 \\
\hline \multicolumn{4}{|c|}{$\begin{array}{l}\text { Sampling days } \\
\text { (median; range) }\end{array}$} \\
\hline & POS & $330(144 ; 383)$ & $359(327 ; 404)$ \\
\hline & Post Vaccine & NA & $24(7 ; 81)$ \\
\hline
\end{tabular}




\section{Article}

Extended Data Table 2 | Characteristics of the cohort of vaccinated individuals

\begin{tabular}{|c|c|c|c|c|}
\hline \multirow[b]{2}{*}{ Orléans cohort: Vaccinated recipients } & & \multirow{2}{*}{$\begin{array}{l}\text { Pfizer } \\
\text { (1 or } 2 \text { doses) } \\
n=16\end{array}$} & \multirow{2}{*}{$\begin{array}{l}\text { AstraZeneca } \\
\text { (1 dose) } \\
n=23\end{array}$} & \multirow{2}{*}{$\begin{array}{l}\text { AstraZeneca } \\
\text { (2 doses) } \\
\mathrm{N}=20\end{array}$} \\
\hline & & & & \\
\hline \multicolumn{5}{|l|}{ Sex } \\
\hline & Female & 5 & 7 & 15 \\
\hline & Male & 11 & 5 & 5 \\
\hline Age (Median; range) & & $60(35 ; 75)$ & $38(28 ; 64)$ & $59.5(55 ; 73)$ \\
\hline Immune deficiency & & 0 & 0 & 0 \\
\hline Previous COVID-19 & & 0 & 0 & 0 \\
\hline Anti-N & & 0 & 0 & 0 \\
\hline 1st dose & & $\operatorname{Jan} 4-8, \quad 2021$ & Feb 8 - April 22, 2021 & Feb 5 - April 7, 2021 \\
\hline 2nd dose & & Jan 20 - Feb 5, 2021 & NA & May $3-19,2021$ \\
\hline \multicolumn{5}{|l|}{$\begin{array}{l}\text { Sampling days post-vaccination } \\
\text { (Median; range) }\end{array}$} \\
\hline & W3 & $20(18 ; 25)$ & & \\
\hline & W8 & $57(53 ; 84)$ & & \\
\hline & W10 & & $70(41 ; 91)$ & \\
\hline & W16 & $118(116 ; 124)$ & & $109(56 ; 116)$ \\
\hline
\end{tabular}




\section{Reporting Summary}

Nature Research wishes to improve the reproducibility of the work that we publish. This form provides structure for consistency and transparency in reporting. For further information on Nature Research policies, see our Editorial Policies and the Editorial Policy Checklist.

\section{Statistics}

For all statistical analyses, confirm that the following items are present in the figure legend, table legend, main text, or Methods section.

n/a Confirmed

$\bigotimes$ The exact sample size $(n)$ for each experimental group/condition, given as a discrete number and unit of measurement

$\square$ \A statement on whether measurements were taken from distinct samples or whether the same sample was measured repeatedly

$\square$ The statistical test(s) used AND whether they are one- or two-sided

$\square$ Only common tests should be described solely by name; describe more complex techniques in the Methods section.

$\square$ \ A description of all covariates tested

$\square$ \A description of any assumptions or corrections, such as tests of normality and adjustment for multiple comparisons

$\square$ A full description of the statistical parameters including central tendency (e.g. means) or other basic estimates (e.g. regression coefficient)

$\bigotimes$ AND variation (e.g. standard deviation) or associated estimates of uncertainty (e.g. confidence intervals)

$\square$ For null hypothesis testing, the test statistic (e.g. $F, t, r$ ) with confidence intervals, effect sizes, degrees of freedom and $P$ value noted

Give $P$ values as exact values whenever suitable.

Х $\square$ For Bayesian analysis, information on the choice of priors and Markov chain Monte Carlo settings

Х $\square$ For hierarchical and complex designs, identification of the appropriate level for tests and full reporting of outcomes

Х $\square$ Estimates of effect sizes (e.g. Cohen's $d$, Pearson's $r$ ), indicating how they were calculated

Our web collection on statistics for biologists contains articles on many of the points above.

\section{Software and code}

Policy information about availability of computer code

Data collection Harmony Software v4.9 (Perkin-Elmer), Attune Nxt Software v3.2.1 (ThermoFischer), Flowjo Software v10.7.1, R v4.1.0, tidyverse v1.3.1, Lubridate v1.7.10

Data analysis Excel 365 v16.46 (Microsoft), Prism v9.0.2 (GraphPad Software)

For manuscripts utilizing custom algorithms or software that are central to the research but not yet described in published literature, software must be made available to editors and reviewers. We strongly encourage code deposition in a community repository (e.g. GitHub). See the Nature Research guidelines for submitting code \& software for further information.

\section{Data}

Policy information about availability of data

All manuscripts must include a data availability statement. This statement should provide the following information, where applicable:

- Accession codes, unique identifiers, or web links for publicly available datasets

- A list of figures that have associated raw data

- A description of any restrictions on data availability

All data are provided as supplementary tables 


\section{Field-specific reporting}

Please select the one below that is the best fit for your research. If you are not sure, read the appropriate sections before making your selection. $\bigotimes$ Life sciences $\quad \square$ Behavioural \& social sciences $\square$ Ecological, evolutionary \& environmental sciences

For a reference copy of the document with all sections, see nature.com/documents/nr-reporting-summary-flat.pdf

\section{Life sciences study design}

All studies must disclose on these points even when the disclosure is negative.

$\begin{array}{ll}\text { Sample size } & \begin{array}{l}162 \text { sera from convalescent, vaccinated and vaccinated convalescent individuals were analyzed in the study. Given the explanatory nature of } \\ \text { the study aiming at describing a phenomenon whose frequency has not yet been established we did not use statistical methods to }\end{array}\end{array}$ predetermine sample size. Thus, we included between 20 and 50 patients per group to allow statistical analysis

Data exclusions None.

Replication All experiments were performed and verified in multiple replicates as indicated in their methods/figure legends.

Randomization The experiments were not randomized as this is not relevant for an observationnal study.

Blinding The investigators were not blinded to allocation as this is not relevant for an observationnal study. However, the clinical sampling and biological measurement were performed by different teams. Only the final assembly of the data revealed the global view of the results.

\section{Reporting for specific materials, systems and methods}

We require information from authors about some types of materials, experimental systems and methods used in many studies. Here, indicate whether each material, system or method listed is relevant to your study. If you are not sure if a list item applies to your research, read the appropriate section before selecting a response.

Materials \& experimental systems

\begin{tabular}{l|l}
\multicolumn{2}{l}{ Methods } \\
\hline n/a & Involved in the study \\
$\square$ & $\square$ ChIP-seq \\
$\square$ & $\bigotimes$ Flow cytometry \\
$\square$ & $\square$ MRI-based neuroimaging
\end{tabular}

n/a Involved in the study

$\square$ \ Antibodies

$\square \bigotimes$ Eukaryotic cell lines

\ $\square$ Palaeontology and archaeology

\ $\square$ MRI-based neuroimaging

$\bigotimes \square$ Animals and other organisms

$\square \bigotimes$ Human research participants

$\square$ \Clinical data

$\bigotimes \mid \square$ Dual use research of concern

\section{Antibodies}

Antibodies used

The anti-S RBD-48, RBD-85, RBD-98, RBD-109, NTD-18, NTD-20, NTD-69 and NTD-71 are human anti-S monoclonal antibodies isolated and produced by Hugo Mouquet (Institut Pasteur). Bamlanivimab, Etesivimab, Casirivimab and Imdevimab are kind gifts of Thierry Prazuck and Laurent Hocqueloux. The Goat anti-Human lgG (H+L) Cross-Adsorbed Secondary Antibody, Alexa Fluor 647 (A21445) was obtained from thermoFisher Scientific.

Validation

The human anti-S RBD-48, RBD-85, RBD-98, RBD-109, NTD-18, NTD-20, NTD-69 and NTD-71 were validated using ELISAs (against the trimeric S, RBD, S2 and NTD proteins) by the team of H.Mouquet. Bamlanivimab, Etesivimab, Casirivimab and Imdevimab were validated by measuring their binding and neutralizing activity against SARS-CoV-2. Validation of the goat anti-human IgG is available from the ThermoFisher website.

\section{Eukaryotic cell lines}

Policy information about cell lines

Cell line source(s)

Vero E6 (ATCC ${ }^{\circledR}$ CRL-1586 $^{\text {TM }}$ ), Freestyle 293-F (ThermoFisher) and U2OS cells (ATCC ${ }^{\circledR}$ HTB-96 ${ }^{\mathrm{TM}}$ ), all obtained from the ATCC.

Authentication

Cell lines were not authenticated.

Mycoplasma contamination

All cells are negative for mycoplasma contamination. Tests are performed on a bi-monthly basis 


\section{Human research participants}

\section{Policy information about studies involving human research participants}

Population characteristics

Orleans' Cohort of convalescent and/or vaccinated individuals: since April 2020, a prospective, monocentric, longitudinal, cohort clinical study enrolling 170 SARS-CoV-2-infected individuals and 59 non-infected healthy controls is on-going, aiming to describe the persistence of specific and neutralizing antibodies over a 24-months period. Relevant co-variates are available in extended table $1 \mathrm{a}$ and 2.

Strasbourg Cohort of convalescent individuals: Since April 2020, a prospective, interventional, monocentric, longitudinal, cohort clinical study enrolling 308 RT-PCR-diagnosed SARS-CoV-2 infected hospital staff from the Strasbourg University Hospitals is on-going. Given the exploratory design of the two studies, the characteristics of participants were not preestablished when entering the cohorts. Relevant co-variates are available in extended table $1 \mathrm{~b}$.

Recruitment

Orléans cohort : Individuals admitted to the hospital for COVID-19 or with known COVID-19 consulting for a chronic disease were invited to participate.

Strasbourg Cohort : Hospital staff with PCR-confirmed COVID-19 were invited to participate.

Individuals were included without any selection other than those imposed by the entry criteria (known COVID-19 or vaccination). Under these conditions, no particular bias is envisaged.

Ethics oversight

Orléans was approved by national external committee (CPP Ile de France IV, IRB No. 00003835). Strasbourg cohort was approved by the institutional review board of Strasbourg University Hospitals. At enrolment a written informed consent was collected for all participants.

Note that full information on the approval of the study protocol must also be provided in the manuscript.

\section{Clinical data}

Policy information about clinical studies

All manuscripts should comply with the ICMJE guidelines for publication of clinical research and a completed CONSORT checklist must be included with all submissions.

Clinical trial registration NCT04750720 and NCT04441684

Study protocol

All protocols can be accessed on clinicaltrial.gov

Data collection

Orléans and strasbourg cohorts started on April 2020 in Strasbourg Hospital (Hopitaux universitaires de Strasbourg) and Orléans Hospital (Centre hospitalier Régional Orléans) respectively, and are on-going.

Outcomes

The primary outcome of both studies was the presence of antibodies to SARS-CoV-2 Spike protein as measured with the S-Flow assay. The secondary outcome was the presence of neutralizing antibodies as measured with the S-Fuse assay.

\section{Flow Cytometry}

\section{Plots}

\section{Confirm that:}

\The axis labels state the marker and fluorochrome used (e.g. CD4-FITC).

\The axis scales are clearly visible. Include numbers along axes only for bottom left plot of group (a 'group' is an analysis of identical markers).

$\bigotimes$ All plots are contour plots with outliers or pseudocolor plots.

$\bigotimes$ A numerical value for number of cells or percentage (with statistics) is provided.

\section{Methodology}

Sample preparation

SARS-CoV-2 infected Vero cells were stained as indicated in the method section. All samples were acquired within $24 \mathrm{~h}$.

Instrument

Attune NxT Acoustic Focusing Cytometer, blue/red/violet/yellow (catalog number : 15360667)

Software

AttuneNxT Software v3.2.1

Cell population abundance

At least 10,000 cells were acquired for each condition.

Gating strategy

All gates were set on uninfected Vero cells.

$\bigotimes$ Tick this box to confirm that a figure exemplifying the gating strategy is provided in the Supplementary Information. 